\title{
1 Ensemble encoding of conditioned fear by prefrontal 2 somatostatin interneurons
}

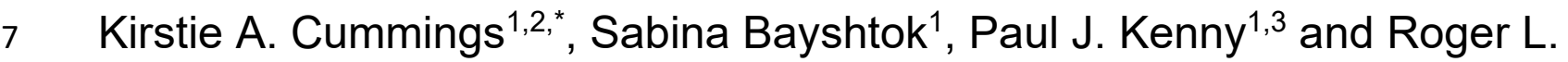

$11{ }^{1}$ Nash Family Department of Neuroscience and the Friedman Brain Institute, Icahn School of

12 Medicine at Mount Sinai, New York, NY

13 2Department of Neurobiology, University of Alabama at Birmingham, Birmingham, AL

14 32Drug Discovery Institute, Icahn School of Medicine at Mount Sinai, New York, NY

15

16

*Correspondence should be addressed to: roger.clem@mssm.edu, kac3@uab.edu.

17

18

19

20 


\section{Abstract}

27 Neurons preferentially activated by learning have been ascribed the unique potential to encode

28 memory. However, it remains unclear which genetically-defined cell types are recruited as part

29 of such an ensemble, or what role discrete subpopulations play in behavior. Here we show that

30 fear conditioning activates a heterogeneous neural ensemble in the medial prefrontal cortex

31 (mPFC), comprised to a large degree of GABAergic interneurons immunoreactive for

32 somatostatin (SST-INs). Using an intersectional genetic approach, we demonstrate that fear

33 learning-activated SST-INs exhibit distinct circuit properties, are preferentially reactivated during

34 memory retrieval, and mediate the expression of defensive freezing. We further show that a

35 rewarding experience, morphine treatment, activates an orthogonal SST-IN population that

36 exerts opposing control over fear. These results outline an important role for discrete

37 GABAergic ensembles in fear memory encoding, and point to an unappreciated capacity for

38 functional specialization among SST-INs. 


\section{Introduction}

Stimuli encountered during learning preferentially activate specific neurons. Studies that monitor and manipulate these populations suggest that they play an essential role in memory encoding and may retain a cellular trace of learning that, when reactivated, mediates memory expression (Bocchio et al., 2017; Clem and Schiller, 2016; Josselyn and Tonegawa, 2020). Despite the theoretical importance of sparse neural coding, the cellular composition of memoryrelated ensembles is largely unexplored, and therefore the functional contribution of specific cell types, defined by gene expression, morphology, or input-output connectivity, remains poorly understood.

The formation of associative fear memory is primarily attributed to glutamatergic projection neurons (PNs), which express a variety of cellular mechanisms for experiencedependent plasticity (Herry and Johansen, 2014; Malenka and Bear, 2004; Ressler and Maren, 2019). However, GABAergic INs also respond to memory-related cues and extensively modulate the function of PNs. In particular, INs containing parvalbumin- (PV-INs), vasoactive intestinal peptide (VIP-INs) and somatostatin (SST-INs) play key roles in orchestrating circuit dynamics underlying memory acquisition and expression (Artinian and Lacaille, 2018; Courtin et al., 2014; Cummings and Clem, 2020; Krabbe et al., 2019; Lucas and Clem, 2017; Wolff et al., 2014; Xu et al., 2019). Synaptic inhibition is thought to facilitate these processes in part through rhythmic entrainment of PN firing (Headley and Pare, 2017). However, evidence suggests that both learning and recall also rely on synaptic interactions between INs that promote PN firing through disinhibition, for example when the recruitment of one IN subtype leads to a corresponding suppression of another (Artinian and Lacaille, 2018; Letzkus et al., 2015; Lucas and Clem, 2018). Because they modulate large groups of PNs, such complex outcomes of GABAergic transmission could endow discrete subsets of INs with unique influence over memory networks. Consequently, an important unanswered question is whether learning recruits specific INs that participate selectively in memory encoding. 
Recently, we demonstrated that SST-INs in the prelimbic cortex exhibit increased synaptic efficacy as well as cue-evoked activity after auditory fear conditioning, suggesting their involvement in memory formation (Cummings and Clem, 2020). We further showed that SSTINs control defensive freezing through activation of a distributed brain network, an effect likely orchestrated through the relief of PV-IN-mediated inhibition. Here, using genetic tagging approaches, we investigate whether SST-INs function in memory encoding as part of a discrete neuronal ensemble. Consistent with a stable cellular representation, prelimbic neurons activated at the time of fear conditioning were essential for subsequent memory expression. Contributing prominently to this heterogeneous population are distinct subset of SST-INs that are preferentially reactivated at memory retrieval and selectively promote defensive freezing.

Conversely, cellular tagging during morphine treatment, a primarily rewarding experience (Jiang et al., 2021; Le Merrer et al., 2009), delineates an orthogonal population of SST-INs that is not only distinct from, but also functionally opposed to fear-encoding SST-INs at the behavioral level.

\section{Results}

\section{Genetic capture of a memory-related ensemble of prefrontal neurons.}

Previous work has identified the dorsomedial mPFC, comprised largely of the prelimbic cortex, as an important site for conditioned stimulus (CS) processing in auditory fear conditioning and a critical substrate for fear expression (Bagur et al., 2021; Burgos-Robles et al., 2009; Corcoran and Quirk, 2007; Courtin et al., 2014; Cummings and Clem, 2020; Dejean et al., 2016; DeNardo et al., 2019; Herry and Johansen, 2014; Karalis et al., 2016; Sotres-Bayon and Quirk, 2010; Xu et al., 2019). To identify neurons selectively recruited by learning in the prelimbic mPFC, we employed a viral genetic approach to permanently tag these cells with a fluorescent reporter.

This strategy relied on expression of an estrogen receptor-dependent Cre-recombinase (ERCreER) under the control of the enhanced synaptic activity responsive element (E-SARE), 
which contains regulatory sequences derived from the arc immediate-early gene (Kawashima et al., 2013). Importantly, recombination of target alleles following induction of ERCreER depends on the presence of the estrogen receptor ligand 4-hydroxytamoxifen (4-OHT), which together with rapid degradation of ERCreER restricts neuronal tagging to a period of several hours following activity.

A viral vector containing the E-SARE-ERCreER construct was co-injected into the mPFC together with vectors encoding a Cre-dependent enhanced yellow fluorescent protein (DIOeYFP) and synapsin-driven mCherry (hSyn-mCherry), the latter of which served purely as a marker of viral infusion (Fig. 1A). Following viral incubation, mice were subjected to auditory fear conditioning, which consisted of paired presentations of an auditory tone $(2 \mathrm{KHz}, 80 \mathrm{~dB}, 20$ s) that co-terminated with an aversive foot shock $(0.7 \mathrm{~mA}, 2 \mathrm{~s})$. As a behavioral control condition, a subset of animals underwent the same procedure, except that aversive foot shocks were omitted (tones only). Following conditioning or tone presentations, subjects received intraperitoneal injections of either 4-OHT or vehicle (Veh) solution and were returned to their home cages for 2 weeks to allow for the expression of recombinant alleles. At this point they were subjected to a test of memory retrieval in a context distinct from the training arena (Fig. S1), followed by cFos immunohistochemistry, to examine the degree to which neurons that were activated during CS-US pairing were specifically reactivated during CS exposure.

Mice injected with 4-OHT after fear conditioning, but not after tones only exposure, exhibited substantially more eYFP-positive neurons than vehicle-treated mice, which contained only sparse background labeling (Fig. 1B, C). Furthermore, while no overall differences in cFos levels were observed following memory retrieval (Fig. 1D), mice that received 4-OHT following fear conditioning exhibited a higher density of double-labeled neurons (Fig. 1E). More importantly, a higher proportion of eYFP-expressing neurons in these animals were immunoreactive for cFos (Fig. 1F), indicating that a greater incidence of double labeling was not attributable to higher levels of eYFP expression. This indicates that neurons activated during 
fear conditioning are preferentially reactivated during memory retrieval, consistent with their selective involvement in long-term memory.

\section{Neurons activated during fear learning control memory expression.}

To test whether learning-activated neurons in the prelimbic cortex mediate memory expression, we next examined whether optogenetic manipulation of these cells influences CSevoked defensive freezing. To enable optogenetic control of these populations, mice received bilateral infusions of a viral cocktail including E-SARE-ERCreER and hSyn-mCherry vectors, along with a vector encoding either a Cre-dependent Archaerhodopsin (FLEX-Arch3.0-GFP; Arch) or channelrhodopsin construct (DIO-ChR2-eYFP; ChR2), and were implanted with optic fibers directed at prelimbic cortex (Fig. 2A, B, D, E). After CS-US pairing, subjects received intraperitoneal injections of either 4-OHT or vehicle and were submitted to a memory retrieval test in which we examined the independent and combined effects of light and CS trials on freezing behavior. Following expression of Arch within learning-activated neurons, photoinhibition (532 nm, $20 \mathrm{~s}$ epoch, constant) resulted in a reduction of freezing during CS trials to levels indistinguishable from baseline (Fig. 2C). Conversely, after ChR2 expression, photoexcitation (473 nm, $20 \mathrm{~s}$ epoch, $20 \mathrm{~Hz}, 5 \mathrm{~ms}$ duration) increased freezing even in the absence of the CS (Fig. 2F). No further increase in freezing was observed when photoexcitation was combined with CS presentation, which could potentially reflect a ceiling effect. Examination of difference scores revealed bidirectional effects of optogenetic manipulations during the baseline period (Fig. 2G), suggesting that a component of the tagged ensemble might be involved in the expression of contextual fear. The same analysis performed for CS trials revealed a decrease in freezing in 4-OHT-injected Arch-expressing animals compared to all other groups (Fig. 2H), suggesting a potential role for the tagged ensemble in cued freezing. Importantly, no light-dependent effects were observed in the vehicle-injected groups, or in 
animals that received 4-OHT after tones only exposure (Fig. S2), indicating that modulation of freezing requires prior conditioning in conjunction with ERCreER activity.

\section{SST-INs are selectively recruited as part of the memory-related ensemble.}

158 We recently demonstrated that CS-US pairing increases CS-related activity of prefrontal SST-

159 INs, and that activation of these cells during conditioning is required for subsequent memory 160 expression (Cummings and Clem, 2020). However, it remains unknown whether SST-INs or other GABAergic subtypes participate in memory storage as part of a sparse ensemble. Collectively, PV-INs, VIP-INs and SST-INs comprise $>80 \%$ of GABAergic neurons in the cortex (Rudy et al., 2011). To determine whether these cell types are recruited into the learning-related ensemble, we performed immunohistochemical staining against markers of these IN subtypes and quantified overlap with an activity-dependent fluorescent tag (Fig. 3). In animals that received fear conditioning in combination with 4-OHT, we observed a far greater density of eYFP-positive SST-INs compared to all other conditions (Fig. 3A-C). In addition, a higher proportion of tagged neurons in this group expressed SST (Fig. 3D), indicating that an increase in labeled SST-INs after conditioning cannot be attributed solely to higher eYFP levels (Fig. 1). Importantly, these effects were not observed after tones only exposure, further indicating that

171 labeling of SST-INs is specific to CS-US pairing and not driven by incidental activity. On

172 average, $\sim 20 \%$ of the tagged ensemble was comprised of SST-INs (Fig. 3D), which in turn

173 represented $\sim 30 \%$ of the total SST-IN population (Fig. 3E).

175 immunoreactivity for PV or VIP, and the incidence of recombination among PV- and VIP-INs

176 was not modulated by conditioning or 4-OHT (Fig. 3F-O). This could potentially indicate that

177 fear conditioning does not strongly activate these cell types or, alternatively, they may exhibit

178 molecular differences that preclude activation and binding of transcription factors CREB, MEF2

179 and SRF to E-SARE in response to behavioral experience (Kawashima et al., 2014). While both 
SST- and VIP-INs respond to aversive foot shocks (Cummings and Clem, 2020; Krabbe et al., 2019; Pi et al., 2013), genetic tagging would require coupling between this type of activity and the above transcriptional responses, which have been linked to memory encoding (Alberini, 2009). Therefore, E-SARE induction potentially demarcates a subset of SST-INs specifically involved in trace formation.

SST-INs activated during fear learning function like an engram-bearing population.

The above results raise the possibility that SST-INs are among the prefrontal neurons activated by learning and subsequently reactivated to mediate memory retrieval (Fig. 1), which are important criteria for engram-bearing cells (Tonegawa et al., 2015). Alternatively, activated SST-INs may be relevant only to initial learning, with recall being mediated by other cell types (e.g. glutamatergic PNs). To discriminate between these possibilities, we made use of intersectional activity-dependent tagging to examine the specific role of learning-activated SSTINs. This strategy resembled the one used for non-selective tagging except that in order to be activated by ERCreER, target vectors were also required to undergo flippase (Flp) recombination, which was restricted to SST-INs by the use of SST-FIpO transgenic mice. Prior to training, these animals received bilateral mPFC injections of a viral cocktail including ESARE-ERCreER and hSyn-mCherry vectors, along with a Cre- and Flp-dependent eYFP construct (Cre on $_{\text {Flp }}$-eYFP) (Fig. 4A). Subjects then underwent auditory fear conditioning, or tones only experience (Fig. S3), and immediately afterwards received intraperitoneal injections of 4-OHT or vehicle. Following a test of memory retrieval in a context distinct from the training arena, we then utilized cFos immunohistochemistry to examine the degree to which SST-INs

202 that were activated during CS-US pairing were reactivated during CS exposure.

Mice that received 4-OHT after fear conditioning, but not tones only exposure, exhibited 204 a higher density of eYFP-positive cells than vehicle controls (Fig. 4B, C). Importantly, we found 205 that a vast majority (>91\%) of neurons labeled in this manner are immunoreactive for SST, 
indicating that our approach captures a relatively pure population of activated SST-INs (Fig.

S4). Although comparable levels of cFos expression were exhibited across conditions (Fig. 4D), conditioning combined with 4-OHT was associated with a higher density of cFos-positive eYFP cells (Fig. 4E) and, importantly, a higher proportion of eYFP cells in these animals exhibited cFos immunoreactivity (Fig. 4F). This pattern of results suggests that cellular activity during memory retrieval is extraordinarily selective for SST-INs that were active at the time of learning. Indeed, nearly $30 \%$ of the tagged SST-IN population was reactivated upon retrieval. conditioning exert control over memory expression. Prior to training, we injected into the mPFC of SST-FlpO transgenic mice a viral cocktail including E-SARE-ERCreER and hSyn-mCherry vectors, along with a Cre- and Flp-dependent ChR2 construct (Cre on $/$ Flpon-ChR2-eYFP) (Fig.

5A-B). Following auditory fear conditioning, or tones only experience, mice received either 4$\mathrm{OHT}$ or vehicle solution and were submitted to a test of memory retrieval in which we examined the independent and combined effects of light and CS trials. Strikingly, selective photoexcitation stimulation of a heterogeneous ensemble (Fig. 2). This was not attributable to non-specific motor effects because photoexcitation did not alter locomotor parameters in the open field test (Fig. S5). In contrast to these results, mice that received tones only experience or unpaired conditioning prior to SST-IN tagging exhibited no change in freezing upon photoexcitation (Fig. S6). These results suggest that fear conditioning activates a subset ( $30 \%)$ of SST-INs that

227 contribute to the storage and expression of cue associations.

Fear memory-encoding SST-INs exhibit unique input and output synaptic transmission. 
232 transmission onto prelimbic SST-INs, and that SST-IN activation at the time of learning is

233 required both for the expression of this plasticity as well as cue-evoked freezing (Cummings and

234 Clem, 2020). However, it remains unclear whether differences in synaptic transmission can

235 explain the preferential reactivation of learning-activated SST-INs. To examine this possibility,

236 we employed an activity-dependent fluorescent tag in conjunction with constitutive labeling of

237 SST-INs, allowing us to measure the synaptic properties of both tagged and non-tagged SST-

238 INs. First, SST-FlpO x Ai65 transgenic mice received bilateral mPFC infusions of E-SARE-

239 ERCreER and Creon/FIpon-eYFP vectors and then underwent fear conditioning followed by 4-

240 OHT injection (Fig. 6A). Electrophysiological recordings were then obtained from learning-

241 activated SST-INs, which were labeled with both eYFP and tdTomato, as well as non-tagged

242 SST-INs, which expressed only tdTomato (Fig. S7). SST-INs activated during learning exhibited

243 a higher frequency of spontaneous excitatory postsynaptic currents (EPSCs) compared to non-

244 tagged SST-INs (Fig. 6B). However, there were no differences in either frequency or amplitude

245 of inhibitory postsynaptic currents (IPSCs) (Fig. 6C). Electrically-evoked EPSCs from tagged

246 SST-INs also exhibited a higher paired-pulse ratio (PPR) compared to those from non-tagged

247 SST-INs, indicating that elevated excitatory transmission onto learning-activated SST-INs is

248 attributable to higher glutamate release probability (Fig. 6D). These differences were not

249 observed when comparing tagged and non-tagged SST-INs from animals that received

250 unpaired training (Fig. S8), suggesting that they are a specific property of cells engaged in cued

251 associative learning, and given our prior findings, they are likely acquired as part of the memory

252 encoding process (Cummings and Clem, 2020). Interestingly, when we examined action

253 potential firing in response to current injection, we also observed higher excitability of tagged

254 versus non-tagged SST-INs after CS-US pairing specifically in male mice (Fig. S9).

255 While increased excitatory input may play a causal role in the reactivation of learning-

256 activated SST-INs, their ability to modulate freezing depends on interaction with other cell types

257 in the mPFC. We previously observed that CS-US pairing, but not unpaired training, alters the 
balance of transmission from prelimbic SST-INs onto PV-INs versus PNs in a manner that favors PN disinhibition (Cummings and Clem, 2020). We therefore sought to determine whether the microcircuit properties of SST-INs specifically engaged by CS-US pairing differ from those of SST-INs activated under cue non-associative, or unpaired, conditions. Specifically, we examined the relative level of GABAergic inhibition that these populations provide onto neighboring PV-INs as well as PNs that were either tagged or non-tagged as a result of training,

264 in order to reveal how SST-INs interact selectively with learning-activated PNs. To accomplish

265 this, we first generated SST-FlpO x PV-Cre $\times$ Ai9 triple transgenic mice, and then bilaterally 266 infused a viral cocktail containing E-SARE-ERCreER and Cre ${ }_{o n} / F I p_{o n}-C h R 2-e Y F P$ vectors into 267 the mPFC of these animals (Fig. 6E). Following fear conditioning and 4-OHT injection, this permitted expression of ChR2-eYFP and tdTomato expression in learning-activated SST-INs and PNs, respectively. In addition, constitutive expression of tdTomato was also driven via the PV-Cre allele within PV-INs, which can be readily distinguished from PNs based on electrophysiological properties.

In acute brain slices, we recorded monosynaptic IPSCs in the above cell populations in response to photoexcitation of learning-activated SST-INs (Fig. 6F). In unpaired mice, responses of tagged and non-tagged PNs were similar in amplitude, and both cell types exhibited larger responses than PV-INs. In contrast, in paired mice, response amplitudes in 276 tagged PNs were smaller than in non-tagged PNs, while those in non-tagged PNs and PV-INs

277 were equivalent (Fig. 6G). To control for potential differences in viral transduction between

278 paired and unpaired animals, IPSCs from PV-INs and tagged PNs were normalized to

279 responses from non-tagged PNs (Fig. 6H). This revealed that compared to unpaired training,

280 SST-INs activated during CS-US pairing provide proportionately stronger input onto PV-INs, and 281 generate weaker responses within tagged PNs. These differences in output, which could 282 potentially result from learning-induced plasticity, may facilitate selective disinhibition of memory-related PNs through concerted mono- and disynaptic control. 


\section{Morphine and fear conditioning recruit functionally discrete SST-IN populations.}

While the above results suggest that memory-encoding SST-INs have distinct circuit properties, it remains possible that freezing elicited by optogenetic stimulation is a non-specific effect of SST-IN transmission, rather than dependent on reactivation of specific cells. In this case, similar effects would be obtained by stimulating other subsets of SST-INs as long as they exceed some threshold population size. Previous work has shown that prelimbic SST-INs exhibit plasticity of membrane and synaptic properties following morphine treatment, an experience highly distinct from fear conditioning (Jiang et al., 2021). We therefore used activity-dependent genetic tagging to investigate whether SST-INs activated by morphine exhibit anatomical overlap with those involved in fear conditioning, and whether reactivation of these cells can drive fear expression after CS-US pairing (Fig. 7; Fig. S10). SST-FIpO transgenic mice received viral infusions identical to those employed for fear-related cellular tagging (Fig. 4) and were then injected with morphine $(10 \mathrm{mg} / \mathrm{kg})$ or saline solution followed 10 hours later by $4-\mathrm{OHT}$ or vehicle (Fig. 7A). Three weeks later, all mice underwent CS-US pairing followed by CS presentation and immunohistochemical staining for cFos to determine the degree to which morphine-activated SST-INs were reactivated by fear memory retrieval. As expected, a higher density of eYFPpositive SST-INs was observed following morphine combined with 4-OHT, relative to control conditions (Fig. 7B-C). Among the groups, however, a comparable proportion of eYFP-positive SST-INs were immunoreactive for cFos, indicating chance levels of overlap between morphinerelated tagging and retrieval-induced activity (Fig. 7D-F). This suggests that in contrast to SSTINs activated during fear conditioning (Fig. 4), morphine-activated SST-INs were not preferentially reactivated by cued memory retrieval.

To examine the impact of morphine-activated SST-INs on fear expression, cellular tagging was performed as above, except that E-SARE-ERCreER was used to drive ChR2-eYFP expression to enable subsequent optogenetic manipulation (Fig. 8A-B). Three weeks later, photoexcitation of morphine tagged SST-INs ( $473 \mathrm{~nm}, 20 \mathrm{~s}$ epochs, $20 \mathrm{~Hz}, 10 \mathrm{~ms}$ pulses) had 
no effect on freezing levels in shock-naïve animals (Fig. 8C). On the next day, all subjects underwent CS-US pairing followed by a test of fear memory retrieval in which we examined the

312 independent and combined effects of light and CS trials. In contrast to stimulation of SST-INs

313 that were activated during fear conditioning (Fig. 5), photoexcitation of morphine-activated SST-

314 INs had no effect on baseline freezing levels. Interestingly, however, when photoexcitation of

315 morphine-activated SST-INs was combined with CS presentation, freezing levels were reduced

316 compared to CS-only trials (Fig. 8D). Importantly, photoexcitation of morphine-activated SST-

317 INs had no effect on open field behavior, implying that reduction of freezing during the test of memory retrieval was not attributable to non-specific locomotor effects (Fig. S11). These results indicate that although both morphine and fear conditioning recruit large populations of SST-INs, they exert distinct behavioral effects. Not only do SST-INs activated during fear conditioning uniquely promote fear expression, but they are functionally opposed by morphine-activated SST-INs.

Discussion

In this study, we demonstrate that a sparse ensemble of neurons in the prelimbic mPFC

326 plays a critical role in fear memory encoding. A major subset of cells activated during learning

327 are SST-INs, whose responsiveness to activity-dependent genetic tagging differentiates them

328 from both PV-INs and VIP-INs. Intersectional genetic capture of learning-activated SST-INs

329 reveals that they are selectively reactivated during memory retrieval and support the expression

330 of defensive freezing, properties that are attributable to differences in transmission within

331 synaptic networks formed by these cells. In particular, memory-related SST-INs receive stronger

332 excitatory input and exhibit biases in GABAergic output that favor disinhibition of PNs recruited

333 by learning. Importantly, fear encoding is a specific property of this cellular subset that is not

334 generalizable to other SST-IN populations. Indeed, SST-INs recruited by a distinct positive

335 experience, morphine exposure, exert opposing control over cued fear expression. 
A likely key to the function of memory-encoding SST-INs is that they interact conditioning, those activated by CS-US pairing give rise to monosynaptic connections onto PV-

INs as well as both activated and non-activated PNs (Fig. 6). However, the balance of

GABAergic transmission onto these populations differed between the training conditions,

reflecting either the recruitment of discrete populations of SST-INs or the expression of plasticity

342 in their output synapses. SST-INs engaged by cued associative learning provide proportionately stronger input onto PV-INs, consistent with previous recordings from our laboratory involving non-selective SST-IN stimulation (Cummings and Clem, 2020). In addition, while SST-INs activated by unpaired training uniformly inhibit PNs, those activated by CS-US pairing provide much weaker inhibition onto activated versus non-activated PNs. The unique network configuration of memory-encoding SST-INs may facilitate PV-IN-mediated disinhibition while simultaneously sparing fear-related PNs from SST-IN-mediated inhibition, enabling recruitment suppression of irrelevant pathways.

In contrast to SST-INs activated by fear conditioning, those labeled in response to additional unexpected finding was that stimulation of morphine-related SST-INs opposes the expression of CS-evoked freezing. It is tempting to speculate based on these results that valence is a functional specialization of these discrete populations or otherwise encoded by

357 these cells through experience-dependent plasticity (Tye, 2018). In either case, representation 358 of positive and negative experiences by different subsets of SST-INs raises the possibility that 359 they recruit distinct PNs through disinhibition. In addition, they may compete for behavioral control through mutually opposing inhibition of these output populations (Garcia-Junco-

361 Clemente et al., 2017). Future work should therefore establish whether morphine-activated 
SST-INs control distinct brain networks or interact directly with cell populations underlying fear memory expression, which may provide unique insights into fear attenuation. neurons, our results identify a sparse cell population in the prelimbic cortex with predicted attributes of a memory engram (Cai et al., 2016; DeNardo et al., 2019; Han et al., 2009; Kim and Cho, 2017; Lacagnina et al., 2019; Liu et al., 2012; Rashid et al., 2016; Reijmers et al., 2007; Ryan et al., 2015; Tayler et al., 2013). While most reports do not explicitly address the cellular composition of engram populations, or focus exclusively on the role of glutamatergic projections, we demonstrate that fear learning activates a heterogeneous ensemble containing GABAergic cells. Not only do activated SST-INs fulfill conventional criteria for engram-bearing neurons, but their stimulation recapitulates the effect of broader ensemble activation. This suggests that a subset of SST-INs can fully orchestrate network dynamics underlying memory retrieval and likely control the reactivation of glutamatergic PNs. Because SST-INs undergo plasticity during learning, our work underscores the importance of establishing how both excitatory and inhibitory neurons contribute to the formation of a cellular memory trace, as well as how these cell types potentially interact to promote memory expression. GABAergic subtypes, functional classification of these cells based on molecular markers is inherently complicated. As one of the most diverse of the broadly defined subtypes, SST-INs have been subclassified based on distinct firing phenotypes, morphologies, and co-expression 382 of secondary markers (Yavorska and Wehr, 2016). For example, while Martinotti cells are 383 estimated to comprise at least half of SST-INs, disinhibition of the thalamorecipient layer of 384 somatosensory cortex is mediated by SST-INs with non-Martinotti morphology (Xu et al., 2013).

385 Within the frontal cortex, functionally relevant subpopulations of SST-INs have been

386 discriminated based on expression of receptors for acetylcholine and oxytocin, as well as 387 signaling components like neuropeptide $Y$ and neuronal nitric oxide synthase (Funk et al., 2017; 
Jackson et al., 2018; Li et al., 2016; Nakajima et al., 2014; Yamamuro et al., 2020). implicated in social and anxiety-related behavior (Li et al., 2016; Nakajima et al., 2014; Yamamuro et al., 2020), which are modulated by the processing of aversive stimuli.

392 Nevertheless, our appreciation of how discrete subpopulations encode different aspects of 393 experience is severely limited. Functional mapping of SST-INs, aided by activity-dependent 394 genetic tagging, may help resolve these questions. underlying associative fear learning are a subset of SST-INs with distinct circuit properties.

397 These cells are preferentially reactivated during memory recall and have a unique capacity to 


\section{References}

Alberini, C.M. (2009). Transcription factors in long-term memory and synaptic plasticity. Physiol Rev 89, 121-145. 10.1152/physrev.00017.2008.

Artinian, J., and Lacaille, J.C. (2018). Disinhibition in learning and memory circuits: New vistas for somatostatin interneurons and long-term synaptic plasticity. Brain Research Bulletin 141, 20-26. 10.1016/j.brainresbull.2017.11.012.

Bagur, S., Lefort, J.M., Lacroix, M.M., de Lavilleon, G., Herry, C., Chouvaeff, M., Billand, C., Geoffroy, H., and Benchenane, K. (2021). Breathing-driven prefrontal oscillations regulate maintenance of conditioned-fear evoked freezing independently of initiation. Nat Commun 12, 2605. 10.1038/s41467021-22798-6.

Bocchio, M., Nabavi, S., and Capogna, M. (2017). Synaptic Plasticity, Engrams, and Network Oscillations in Amygdala Circuits for Storage and Retrieval of Emotional Memories. Neuron 94, 731-743. 10.1016/j.neuron.2017.03.022.

Burgos-Robles, A., Vidal-Gonzalez, I., and Quirk, G.J. (2009). Sustained conditioned responses in prelimbic prefrontal neurons are correlated with fear expression and extinction failure. J Neurosci 29, 8474-8482. 10.1523/JNEUROSCI.0378-09.2009.

Cai, D.J., Aharoni, D., Shuman, T., Shobe, J., Biane, J., Song, W., Wei, B., Veshkini, M., La-Vu, M., Lou, J., et al. (2016). A shared neural ensemble links distinct contextual memories encoded close in time. Nature $534,115-118$.

Clem, R.L., and Schiller, D. (2016). New Learning and Unlearning: Strangers or Accomplices in Threat Memory Attenuation? Trends Neurosci 39, 340-351. 10.1016/j.tins.2016.03.003.

Corcoran, K.A., and Quirk, G.J. (2007). Activity in prelimbic cortex is necessary for the expression of learned, but not innate, fears. J Neurosci 27, 840-844. 10.1523/JNEUROSCI.5327-06.2007.

Courtin, J., Chaudun, F., Rozeske, R.R., Karalis, N., Gonzalez-Campo, C., Wurtz, H., Abdi, A., Baufreton, J., Bienvenu, T.C., and Herry, C. (2014). Prefrontal parvalbumin interneurons shape neuronal activity to drive fear expression. Nature 505, 92-96. 10.1038/nature12755.

Cummings, K.A., and Clem, R.L. (2020). Prefrontal somatostatin interneurons encode fear memory. Nat Neurosci 23, 61-74. 10.1038/s41593-019-0552-7.

Dejean, C., Courtin, J., Karalis, N., Chaudun, F., Wurtz, H., Bienvenu, T.C., and Herry, C. (2016). Prefrontal neuronal assemblies temporally control fear behaviour. Nature 535, 420-424. 10.1038/nature18630.

DeNardo, L.A., Liu, C.D., Allen, W.E., Adams, E.L., Friedmann, D., Fu, L., Guenthner, C.J., Tessier-Lavigne, M., and Luo, L. (2019). Temporal evolution of cortical ensembles promoting remote memory retrieval. Nat Neurosci 22, 460-469. 10.1038/s41593-018-0318-7.

Funk, C.M., Peelman, K., Bellesi, M., Marshall, W., Cirelli, C., and Tononi, G. (2017). Role of SomatostatinPositive Cortical Interneurons in the Generation of Sleep Slow Waves. J Neurosci 37, 9132-9148. 10.1523/JNEUROSCI.1303-17.2017.

Garcia-Junco-Clemente, P., Ikrar, T., Tring, E., Xu, X., Ringach, D.L., and Trachtenberg, J.T. (2017). An inhibitory pull-push circuit in frontal cortex. Nat Neurosci 20, 389-392. 10.1038/nn.4483.

Han, J.H., Kushner, S.A., Yiu, A.P., Hsiang, H.L., Buch, T., Waisman, A., Bontempi, B., Neve, R.L., Frankland, P.W., and Josselyn, S.A. (2009). Selective erasure of a fear memory. Science 323, 1492-1496. 10.1126/science.1164139. 
Headley, D.B., and Pare, D. (2017). Common oscillatory mechanisms across multiple memory systems. NPJ Sci Learn 2. 10.1038/s41539-016-0001-2.

Herry, C., and Johansen, J.P. (2014). Encoding of fear learning and memory in distributed neuronal circuits. Nat Neurosci 17, 1644-1654. 10.1038/nn.3869.

Jackson, J., Karnani, M.M., Zemelman, B.V., Burdakov, D., and Lee, A.K. (2018). Inhibitory Control of Prefrontal Cortex by the Claustrum. Neuron 99, 1029-1039 e1024. 10.1016/j.neuron.2018.07.031.

Jiang, C., Wang, X., Le, Q., Liu, P., Liu, C., Wang, Z., He, G., Zheng, P., Wang, F., and Ma, L. (2021). Morphine coordinates SST and PV interneurons in the prelimbic cortex to disinhibit pyramidal neurons and enhance reward. Mol Psychiatry 26, 1178-1193. 10.1038/s41380-019-0480-7.

Josselyn, S.A., and Tonegawa, S. (2020). Memory engrams: Recalling the past and imagining the future. Science 367, eaaw4325-eaaw4325.

Karalis, N., Dejean, C., Chaudun, F., Khoder, S., Rozeske, R.R., Wurtz, H., Bagur, S., Benchenane, K., Sirota, A., Courtin, J., and Herry, C. (2016). 4-Hz oscillations synchronize prefrontal-amygdala circuits during fear behavior. Nat Neurosci 19, 605-612. 10.1038/nn.4251.

Kawashima, T., Kitamura, K., Suzuki, K., Nonaka, M., Kamijo, S., Takemoto-Kimura, S., Kano, M., Okuno, H., Ohki, K., and Bito, H. (2013). Functional labeling of neurons and their projections using the synthetic activity-dependent promoter E-SARE. Nat Methods 10, 889-895. 10.1038/nmeth.2559.

Kawashima, T., Okuno, H., and Bito, H. (2014). A new era for functional labeling of neurons: activitydependent promoters have come of age. Front Neural Circuits 8, 37. 10.3389/fncir.2014.00037.

Kim, W.B., and Cho, J.H. (2017). Encoding of Discriminative Fear Memory by Input-Specific LTP in the Amygdala. Neuron 95, 1129-1146 e1125. 10.1016/j.neuron.2017.08.004.

Krabbe, S., Paradiso, E., d'Aquin, S., Bitterman, Y., Courtin, J., Xu, C., Yonehara, K., Markovic, M., Muller, C., Eichlisberger, T., et al. (2019). Adaptive disinhibitory gating by VIP interneurons permits associative learning. Nat Neurosci 22, 1834-1843. 10.1038/s41593-019-0508-y.

Lacagnina, A.F., Brockway, E.T., Crovetti, C.R., Shue, F., McCarty, M.J., Sattler, K.P., Lim, S.C., Santos, S.L., Denny, C.A., and Drew, M.R. (2019). Distinct hippocampal engrams control extinction and relapse of fear memory. Nature Neuroscience 22, 753-761. 10.1038/s41593-019-0361-z.

Le Merrer, J., Becker, J.A., Befort, K., and Kieffer, B.L. (2009). Reward processing by the opioid system in the brain. Physiol Rev 89, 1379-1412. 10.1152/physrev.00005.2009.

Letzkus, J.J., Wolff, S.B., and Luthi, A. (2015). Disinhibition, a Circuit Mechanism for Associative Learning and Memory. Neuron 88, 264-276. 10.1016/j.neuron.2015.09.024.

Li, K., Nakajima, M., Ibanez-Tallon, I., and Heintz, N. (2016). A Cortical Circuit for Sexually Dimorphic Oxytocin-Dependent Anxiety Behaviors. Cell 167, 60-72 e11. 10.1016/j.cell.2016.08.067.

Liu, X., Ramirez, S., Pang, P.T., Puryear, C.B., Govindarajan, A., Deisseroth, K., and Tonegawa, S. (2012). Optogenetic stimulation of a hippocampal engram activates fear memory recall. Nature 484, 381-385.

Lucas, E.K., and Clem, R.L. (2017). GABAergic interneurons_The orchestra or the conductor in fear learning and memory? Brain Research Bulletin, 0-1.

Lucas, E.K., and Clem, R.L. (2018). GABAergic interneurons: The orchestra or the conductor in fear learning and memory? Brain Res Bull 141, 13-19. 10.1016/j.brainresbull.2017.11.016. 
Malenka, R.C., and Bear, M.F. (2004). LTP and LTD: an embarrassment of riches. Neuron 44, 5-21. 10.1016/j.neuron.2004.09.012.

Nakajima, M., Gorlich, A., and Heintz, N. (2014). Oxytocin modulates female sociosexual behavior through a specific class of prefrontal cortical interneurons. Cell 159, 295-305. 10.1016/j.cell.2014.09.020.

Pi, H.-J., Hangya, B., Kvitsiani, D., Sanders, J.I., Huang, Z.J., and Kepecs, A. (2013). Cortical interneurons that specialize in disinhibitory control. Nature 503, 521-524.

Rashid, A.J., Yan, C., Mercaldo, V., Hsiang, H.L., Park, S., Cole, C.J., De Cristofaro, A., Yu, J., Ramakrishnan, C., Lee, S.Y., et al. (2016). Competition between engrams influences fear memory formation and recall. Science 353, 383-387. 10.1126/science.aaf0594.

Reijmers, L.G., Perkins, B.L., Matsuo, N., and Mayford, M. (2007). Localization of a stable neural correlate of associative memory. Science 317, 1230-1233. 10.1126/science.1143839.

Ressler, R.L., and Maren, S. (2019). Synaptic encoding of fear memories in the amygdala. Curr Opin Neurobiol 54, 54-59. 10.1016/j.conb.2018.08.012.

Rudy, B., Fishell, G., Lee, S., and Hjerling-Leffler, J. (2011). Three groups of interneurons account for nearly $100 \%$ of neocortical GABAergic neurons. Dev Neurobiol 71, 45-61. 10.1002/dneu.20853.

Ryan, T.J., Roy, D.S., Pignatelli, M., Arons, A., and Tonegawa, S. (2015). Memory. Engram cells retain memory under retrograde amnesia. Science 348, 1007-1013. 10.1126/science.aaa5542.

Sotres-Bayon, F., and Quirk, G.J. (2010). Prefrontal control of fear: more than just extinction. Curr Opin Neurobiol 20, 231-235. 10.1016/j.conb.2010.02.005.

Tayler, K.K., Tanaka, K.Z., Reijmers, L.G., and Wiltgen, B.J. (2013). Reactivation of neural ensembles during the retrieval of recent and remote memory. Current Biology 23, 99-106. 10.1016/j.cub.2012.11.019.

Tonegawa, S., Liu, X., Ramirez, S., and Redondo, R. (2015). Memory Engram Cells Have Come of Age. Neuron 87, 918-931. 10.1016/j.neuron.2015.08.002.

Tye, K.M. (2018). Neural Circuit Motifs in Valence Processing. Neuron 100, 436-452. 10.1016/j.neuron.2018.10.001.

Wolff, S.B., Grundemann, J., Tovote, P., Krabbe, S., Jacobson, G.A., Muller, C., Herry, C., Ehrlich, I., Friedrich, R.W., Letzkus, J.J., and Luthi, A. (2014). Amygdala interneuron subtypes control fear learning through disinhibition. Nature 509, 453-458. 10.1038/nature13258.

Xu, H., Jeong, H.Y., Tremblay, R., and Rudy, B. (2013). Neocortical somatostatin-expressing GABAergic interneurons disinhibit the thalamorecipient layer 4. Neuron 77, 155-167.

10.1016/j.neuron.2012.11.004.

Xu, H., Liu, L., Tian, Y., Wang, J., Li, J., Zheng, J., Zhao, H., He, M., Xu, T.L., Duan, S., and Xu, H. (2019). A Disinhibitory Microcircuit Mediates Conditioned Social Fear in the Prefrontal Cortex. Neuron 102, 668682 e665. 10.1016/j.neuron.2019.02.026.

Yamamuro, K., Bicks, L.K., Leventhal, M.B., Kato, D., Im, S., Flanigan, M.E., Garkun, Y., Norman, K.J., Caro, K., Sadahiro, M., et al. (2020). A prefrontal-paraventricular thalamus circuit requires juvenile social experience to regulate adult sociability in mice. Nat Neurosci 23, 1240-1252. 10.1038/s41593-020-06956. 
534 Yavorska, I., and Wehr, M. (2016). Somatostatin-Expressing Inhibitory Interneurons in Cortical Circuits.

535 Front Neural Circuits 10, 76. 10.3389/fncir.2016.00076.

536

537 Author contributions

538 K.A.C. and R.L.C. initiated the project; R.L.C. and P.J.K. supervised research; K.A.C. and

539 R.L.C. designed experiments; K.A.C. and S.B. performed the research and data analysis;

540 R.L.C. and K.A.C. wrote the manuscript.

541

542 Acknowledgments

543 We thank Anosha Khawaja for animal care, Haruhiko Bito for sharing the E-SARE-ERCreER

544 plasmid, and members of the Clem laboratory for helpful discussions. These experiments were

545 supported by funds from the National Institute of Mental Health (NIMH) grants R01 MH116145

546 and R21 MH114170 to R.L.C., in addition to F32 MH115688 and K99 MH122228 to K.A.C.

547

548 Competing interests

549 P.J.K. is co-founder and shareholder in Eolas Therapeutics Inc., which has a licensing

550 agreement with AstraZeneca to develop a novel therapeutic for the treatment of substance use

551 disorders.

552

553

554

555

556

557

558 


\section{Figure 1. Fear conditioning recruits a prefrontal ensemble that is reactivated upon}

retrieval. (A) Wildtype (C57BI/6J) mice received bilateral infusions of a cocktail containing vectors encoding E-SARE-ERCreER, Cre-dependent eYFP, and hSyn-mCherry in mPFC. Four weeks later, mice were either subjected to auditory tones (tones only) or CS-US pairing (conditioning) and then immediately injected with vehicle (veh) or 4-hydroxytamoxifen (4-OHT).

566 Two weeks later, in a neutral context, all mice underwent a test of CS-evoked memory retrieval

567 followed by cFos immunohistochemical analysis. (B) Top: representative histological images of eYFP tagging. Scale bar $=500 \mu \mathrm{m}$. Bottom: Induction of cFos following the retrieval test. White arrowheads denote eYFP tagged neurons immunoreactive for cFos. Scale bar $=100 \mu \mathrm{m} . \mathrm{Cg} 1=$ cingulate area 1. $\mathrm{PL}=$ prelimbic cortex. IL = infralimbic cortex. $(\mathbf{C}-\mathbf{F})$ Comparison between tones only vehicle ( $n=8$ mice), tones only $4-\mathrm{OHT}(n=7$ mice), conditioned vehicle $(n=8$ mice), and conditioned 4-OHT ( $n=7$ mice) groups of (C) number of eYFP+ cells: $\chi^{2}=23.69(3), p=2.89 x$ $10^{-5}$, Kruskal-Wallis ANOVA; (D) number of cFos+ cells: $\chi^{2}=5.75(3), p=0.12$, Kruskal-Wallis

574 ANOVA; (E) number of cFos+/eYFP+ double positive cells: $\chi^{2}=20.91(3), p=1.09 \times 10^{-4}$,

575 Kruskal-Wallis ANOVA; and (F) number of cFos+/eYFP+ double positive cells normalized to the total number of eYFP+ cells: $\chi^{2}=16.73(3), p=8.05 \times 10^{-4}$, Kruskal-Wallis ANOVA. Experiment was performed in 3 different cohorts and pooled together. ${ }^{*} p<0.05,{ }^{* *} p<0.01,{ }^{* * *} p<0.001$, Dunn's post hoc test (C-F).

Figure 2. Prefrontal neurons activated by fear learning mediate conditioned freezing. (A)

581 For in vivo optogenetic silencing of fear learning-related neurons, wildtype mice received

582 bilateral infusions of a cocktail of vectors encoding E-SARE-ERCreER, Cre-dependent Arch, 583 and hSyn-mCherry and were implanted with optic ferrules aimed at PL. Mice were subjected to 584 CS-US pairing and immediately injected with vehicle (veh) or 4-hydroxytamoxifen (4-OHT). 
Freezing was quantified two weeks later in a neutral context in response to independent and combined light and CS trials. (B) Representative histological images of Arch expression and optic fiber placement. Scale $=500 \mu \mathrm{m}$. (C) Modulation of freezing by light $(532 \mathrm{~nm}$ solid light, 20 s epochs) and CS trials in vehicle (gray) and 4-OHT (purple) injected mice. Vehicle: $\mathrm{F}_{(1,8)}=$ 408.43, $p=3.75 \times 10^{-8}, 1$-way repeated measures ANOVA, $n=9$ mice. 4-OHT: $\chi^{2}=27.84(3), p$ $=3.92 \times 10^{-6}$, Friedman ANOVA, $n=10$ mice. Experiments were performed in 3 different cohorts and pooled together. (D) For in vivo optogenetic activation of fear learning-related neurons, wildtype mice received bilateral infusions of a cocktail of vectors encoding E-SAREERCreER, Cre-dependent ChR2, and hSyn-mCherry and were implanted with optic ferrules aimed at PL. Behavior was conducted in a manner identical to (A). (E) Representative histological images of ChR2 expression and optic fiber placement. Scale $=500 \mu \mathrm{m}$. (F) Modulation of freezing by light $(473 \mathrm{~nm}, 5 \mathrm{~ms}$ pulses, $20 \mathrm{~Hz}, 20 \mathrm{~s}$ epochs $)$ and CS trials in vehicle (gray) and 4-OHT (purple) injected mice. Vehicle: $F_{(1,8)}=269.4, p=1.91 \times 10^{-7}$, 1-way repeated measures ANOVA, $n=9$ mice. 4-OHT: $F_{(1,15)}=196.72, p=4.99 \times 10^{-10}$, 1-way repeated measures ANOVA, $n=16$ mice. Experiments were performed in 4 different cohorts and pooled together. (G) Change in freezing induced by photostimulation during the baseline

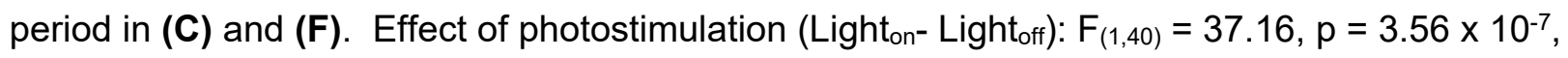
interaction between opsin and light, 2-way ANOVA. (H) Change in freezing induced by photostimulation during CS trials in (C) and (F). Effect of photostimulation (Lighton- Light ${ }_{\text {off }}$ ): $F_{(1,40)}=14.45, p=4.8 \times 10^{-4}$, interaction between opsin and light, 2-way ANOVA. * $p<0.05,{ }^{* *} p$ $<0.01,{ }^{* * *} p<0.001$, Tukey's post-hoc test (c: vehicle, F, G); ${ }^{*} p<0.05,{ }^{* *} p<0.01,{ }^{* *} p<$ 0.001, Dunn's post-hoc test (c: $4-\mathrm{OHT}) . \mathrm{Cg} 1$ = cingulate area $1 . \mathrm{PL}=$ prelimbic cortex. IL = infralimbic cortex.

\section{Figure 3. Fear learning-related ensembles are partly comprised of somatostatin-}

expressing interneurons. (A) Immunohistochemical staining against somatostatin (SST) in 
611 prelimbic cortex tissue following CS-US pairing and 4-OHT injection. Scale $=50 \mu \mathrm{m}$. (B-E)

612 Comparison between tones only vehicle $(n=8$ mice $)$, tones only 4-OHT $(n=8$ mice),

613 conditioning vehicle ( $n=8$ mice), and conditioning 4-OHT ( $n=7$ mice) groups of (B) SST+ cells,

$614 \chi^{2}=6.934(3), p=0.074$, Kruskal-Wallis ANOVA; (C) SST+ eYFP cells, $\chi^{2}=16.9(3), p=7.4 x$

$61510^{-4}$, Kruskal-Wallis ANOVA; (D) SST+ eYFP cells normalized to the total number of eYFP,

$616 \mathrm{~F}_{(1,27)}=87.91, \mathrm{p}=5.55 \times 10^{-10}$, interaction between training and treatment, 2-way ANOVA, and

617 (E) SST+ eYFP cells normalized to the total number of SST + cells per group, $\chi^{2}=17.04(3), p=$

$6186.93 \times 10^{-4}$, Kruskal-Wallis ANOVA. (F) Immunohistochemical staining against parvalbumin (PV)

619 in prelimbic cortex tissue following CS-US pairing and 4-OHT injection. Scale = $50 \mu \mathrm{m}$. (G-J)

620 Comparison between tones only vehicle $(n=3)$, tones only 4-OHT $(n=4)$, conditioning vehicle

$621(n=4)$, and conditioning 4-OHT $(n=4)$ groups of $(\mathbf{G}) \mathrm{PV}+$ cells, $F_{(1,40)}=0.73, p=0.41,2-$ way

622 ANOVA; (H) PV+ eYFP cells, $\chi^{2}=0.485$ (3), $p=0.922$, Kruskal-Wallis ANOVA; (I) PV+ eYFP

623 cells normalized to the total number of eYFP+ cells per group, $\chi^{2}=0.882(3), p=0.829$,

624 Kruskal-Wallis ANOVA; and (J) PV+ eYFP cells normalized to the total number of PV+ cells per

625 group, $\chi^{2}=1.31(3), p=0.727$, Kruskal-Wallis ANOVA. (K) Immunohistochemical staining

626 against vasoactive intestinal peptide (VIP) in prelimbic cortex tissue following CS-US pairing

627 and 4-OHT injection. Scale $=50 \mu \mathrm{m}$. (L-O) Comparison between tones only vehicle $(n=8)$,

628 tones only 4-OHT $(n=8)$, conditioning vehicle $(n=8)$, and conditioning 4-OHT $(n=8)$ groups of

629 (L) VIP+ cells, $\chi^{2}=1.84(3), p=0.606$, Kruskal-Wallis ANOVA; (M) VIP+ eYFP cells, $\chi^{2}=1.56$

630 (3), $p=0.668$, Kruskal-Wallis ANOVA; (N) VIP+ eYFP cells normalized to the total number of

631 eYFP+ cells per group, $\chi^{2}=0.359(3), p=0.949$, Kruskal-Wallis ANOVA; and (O) VIP+ eYFP

632 cells normalized to the total number of VIP+ cells per group, $\chi^{2}=1.02(3), p=0.796$, Kruskal-

633 Wallis ANOVA. Experiments were performed in 2 different cohorts and pooled together. ${ }^{* * *} p<$

634 0.001, Tukey's post-hoc test (D). ${ }^{*} p<0.05,{ }^{* *} p<0.01,{ }^{* * *} p<0.001$, Dunn's post-hoc test

635 (C,E). 

prelimbic cortex of a cocktail containing vectors encoding E-SARE-ERCreER, Cre- and Flpdependent eYFP, and hSyn-mCherry. Four weeks later, mice received either CS-US pairing (conditioning) or auditory tones (tones only) and were then immediately injected with vehicle

(veh) or 4-hydroxytamonifen (4-OHT). After an additional three weeks, in a neutral context, all mice underwent a test of CS-evoked memory retrieval followed by cFos immunohistochemical analysis. (B) Top: representative histological images of eYFP tagging. Scale bar $=500 \mu \mathrm{m}$. immunoreactive for cFos. Scale bar $=100 \mu \mathrm{m} . \mathrm{Cg} 1=$ cingulate area $1 . \mathrm{PL}=$ prelimbic cortex. IL = infralimbic cortex. (C-F) Comparison between tones only vehicle $(n=6$ mice $)$, tones only 4cFos+/eYFP+ double positive cells normalized to the total number of eYFP+ cells in each group: $\chi^{2}=17.53(3), p=5.49 \times 10^{-4}$, Kruskal-Wallis ANOVA. Experiment was performed in 4 different cohorts and pooled together. ${ }^{*} p<0.05,{ }^{* *} p<0.01,{ }^{* * *} p<0.001$, Dunn's post-hoc test (C,E,F). 
661

662

663

664

665

666

667

668

669

670

671

672

673

674

675

676

677

678

679

680

681

682

683

684

685

686

neutral context while testing the independent and combined effect of light and CS trials. (B) Representative histological images of ChR2 expression and optic fiber placement. Scale $=500$ $\mu \mathrm{m} . \mathrm{Cg} 1=$ cingulate area $1 . \mathrm{PL}=$ prelimbic cortex. $\mathrm{IL}=$ infralimbic cortex. $(\mathbf{C})$ Modulation of freezing by light ( $473 \mathrm{~nm}, 10 \mathrm{~ms}$ pulses, $20 \mathrm{~Hz}, 20 \mathrm{~s}$ epochs) and CS trials in vehicle (gray) and 4-OHT (purple) injected mice. Vehicle: $\chi^{2}=19.5$ (3), $p=2.15 \times 10^{-4}$, Friedman ANOVA, $\mathrm{n}=8$ mice. 4-OHT: $\mathrm{F}_{(3,18)}=46.7, \mathrm{p}=1.07 \times 10^{-8}$, 1-way repeated measures ANOVA, $\mathrm{n}=7$ mice. Experiment was performed in 2 different cohorts and pooled together. ${ }^{* *} p<0.01,{ }^{* * *} p<0.001$, Tukey's post-hoc test (C: 4-OHT). * $\mathrm{p}<0.05$, Dunn's post-hoc test (C: vehicle).

\section{Figure 6: Input and output synaptic connections of learning-activated somatostatin}

interneurons exhibit distinct properties. (A) To independently target and record from both tagged and non-tagged SST-INs, which represent activated and non-activated populations, we infused into the prelimbic cortex of SST-FIpO/ Ai65F double transgenic mice a cocktail of vectors encoding E-SARE-ERCreER, as well as Cre- and Flp-dependent eYFP. Mice were subjected to CS-US pairing and immediately injected with 4-hydroxytamoxifen (4-OHT). Three weeks later, recordings were obtained from eYFP+/ tdTomato+ (tagged) and eYFP-/ tdTomato+ (non-tagged) SST-INs. (B) Spontaneous excitatory postsynaptic currents (EPSCs) were collected by interleaved recordings from tagged $(n=15$ cells) and non-tagged SST-INs $(n=15$ cells) in the same slices ( $n=6$ slices from 5 mice). Interevent interval: $U=205, p=1.35 \times 10^{-4}$, Mann-Whitney U-test. Amplitude: $t_{28}=-1.03, p=0.31$, two-sided unpaired t-test. (C) Spontaneous inhibitory postsynaptic currents (IPSCs) were recorded from tagged ( $\mathrm{n}=14$ cells) and non-tagged SST-INs ( $n=14$ cells) in the same slices $(n=6$ slices from 5 mice). Interevent interval: $U=89, p=0.696$, Mann-Whitney U-test. Amplitude: $t_{26}=0.945, p=0.353$, MannWhitney U-test. (D) EPSC recordings from tagged $(n=10$ cells) and non-tagged SST-INs $(n=$ 11 cells) in the same slices ( $n=4$ slices in 3 mice) during paired pulse stimulation. Paired pulse ratio: $F_{(3,7)}=16.64, p=0.0014$, interaction between cell type and delay, 2-way repeated 
measures ANOVA. (E) To isolate GABAergic responses at connections from tagged SST-INs onto PV-INs as well as tagged and non-tagged PNs, SST-FIpO/ PV-IRES-Cre/ Ai9 triple transgenic mice received infusions of a cocktail of vectors encoding E-SARE-ERCreER, as well as Cre- and Flp-dependent ChR2. Mice were subjected to either CS-US pairing or unpaired conditioning and injected with 4-OHT. Three weeks later, recordings were obtained from

692 tdTomato+ PV-INs as well as tagged (tdTomato+) and non-tagged (td-Tomato-) PNs. (F)

693 Example IPSC traces. (G) Amplitude of IPSCs resulting from photoexcitation (460 nm, $1 \mathrm{~ms}, 0.1$ $694 \mathrm{~Hz}$ ) of tagged SST-INs in PV-INs, non-tagged PNs (NT-PNs), and tagged PNs (T-PNs) following unpaired conditioning ( $n=3$ slices from 3 mice: PV-INs ( $n=10$ cells), NT-PNs $(n=10$ cells), T-

696 PNs ( $n=8$ cells $)$ ) and CS-US pairing ( $n=3$ slices from 3 mice: PV-INs $(n=9$ cells), NT-PNs ( $n$ $697=14$ cells), T-PNs ( $n=12$ cells)). Unpaired conditioning: $\chi^{2}=12.95(2), p=0.0015$, Kruskalamplitudes of PV-INs and tagged PNs normalized to the median amplitude of non-tagged PNs from the same slices. PV-IN amplitude: $t_{4}=3.56, p=0.023$, two-sided unpaired $t$-test. Tagged PN amplitude: $t_{4}=4.19, p=0.013$, two-sided unpaired t-test. ${ }^{*} p<0.05,{ }^{* *} p<0.01,{ }^{* * *} p<$ 0.001 by Mann Whitney U-test (B), Tukey's post-hoc test (D), unpaired t-test $(\mathbf{H})$ or Dunn's post703 hoc test (G).

Figure 7: Morphine treatment activates a population of somatostatin-interneurons received bilateral infusions into prelimbic cortex of a cocktail containing vectors encoding $\mathrm{E}$ SARE-ERCreER, Cre- and Flp-dependent eYFP, and hSyn-mCherry. Four weeks later, in the home cage, they were injected with saline or morphine $(10 \mathrm{mg} / \mathrm{kg})$ followed 10 hours later by injections of vehicle (veh) or 4-hydroxytamoxifen (4-OHT). After another 3 weeks, they 
retrieval and cFos immunochemical analysis. (B) Top: representative histological images of SST-IN tagging for mice injected with saline and morphine and subsequently injected with either vehicle or 4-OHT. Scale bar $=500 \mu \mathrm{m}$. Bottom: Induction of cFos following CS-evoked memory retrieval. White arrowhead denotes cFos + tagged SST-IN. Scale bar $=100 \mu \mathrm{m} . \mathrm{Cg} 1=$ cingulate area 1. $\mathrm{PL}=$ prelimbic cortex. IL = infralimbic cortex. $(\mathbf{C}-\mathbf{F})$ Comparison between saline vehicle ( $n=6$ mice), tones only 4-OHT ( $n=6$ mice), conditioning vehicle ( $n=6$ mice), and conditioning 4-OHT ( $n=6$ mice) groups of $(C)$ number of eYFP+ cells: $F_{(1,20)}=96.09, p=4.418 \times 10^{-9}$, interaction between drug and treatment, 2-way ANOVA; (D) number of cFos+ puncta: $F_{(1,20)}=$ 2.62, $p=0.121$, 2-way ANOVA; $(E)$ number of cFos+/eYFP+ double positive cells: $F_{(1,20)}=7.77$, $p=0.011$, interaction between drug and treatment, 2-way ANOVA; and $(\mathbf{F})$ number of cFos+/eYFP+ double positive cells normalized to the total number of eYFP+ cells in each group: $\chi^{2}=4.95$ (3), $p=0.175$, Kruskal-Wallis ANOVA. ${ }^{* *} p<0.01,{ }^{* *} p<0.001$, Tukey's post-hoc test $(\mathbf{C}, \mathbf{E})$.

Figure 8: Somatostatin-interneurons activated by morphine oppose conditioned fear expression. (A) For in vivo optogenetic activation of morphine-related SST-INs, SST-FIpO transgenic mice received bilateral infusions into prelimbic cortex of a cocktail of vectors encoding E-SARE-ERCreER, Cre- and Flp-dependent ChR2, and hSyn-mCherry and were implanted with optic ferrules aimed at PL. Mice received an injection of morphine $(10 \mathrm{mg} / \mathrm{kg})$ and 10 hours later, injections of vehicle (veh) or 4-hydroxytamoxifen (4-OHT). Photostimulationinduced freezing (Stimulation) was quantified three weeks later in a neutral context. Mice were then subjected to CS-US pairing (Conditioning) and 24 hours later, a CS-evoked memory retrieval test (Retrieval) in a neutral context, where the independent and combined effects of light and CS trials were quantified. (B) Representative histological images of ChR2 expression and optic fiber placement. Scale $=500 \mu \mathrm{m} . \mathrm{Cg} 1=$ cingulate area $1 . \mathrm{PL}=$ prelimbic cortex. $\mathrm{IL}=$ infralimbic cortex. (C) Modulation of freezing by photoexcitation (473 nm, $10 \mathrm{~ms}$ pulses, $20 \mathrm{~Hz}$, 
73820 s epochs) in vehicle (gray) and 4-OHT (purple) mice. Vehicle: $t_{7}=-0.788, p=0.456$, paired

739 t-test. 4-OHT: $t_{8}=-1.74, p=0.119$, paired t-test. (D) Modulation of freezing by photoexcitation

740 (473 nm, $10 \mathrm{~ms}$ pulses, $20 \mathrm{~Hz}, 20$ s epochs) and CS presentation during the retrieval test for

741 vehicle (gray) and 4-OHT (purple) mice. Vehicle: $F_{(3,21)}=109.48, p=5.54 \times 10^{-13}, 1$-way

742 repeated measures ANOVA. 4-OHT: $F_{(3,24)}=46.69, p=3.59 \times 10^{-10}, 1$-way repeated measures

743 ANOVA. ${ }^{* *} p<0.01,{ }^{* *} p<0.001$, Tukey's post-hoc test (D). 
bioRxiv preprint doi: https://doi.org/10.1101/2021.07.18.452791; this version posted July 19, 2021. The copyright holder for this preprint (which was not certified by peer review) is the author/funder, who has granted bioRxiv a license to display the preprint in perpetuity. It is made available under aCC-BY-NC-ND 4.0 International license.

A
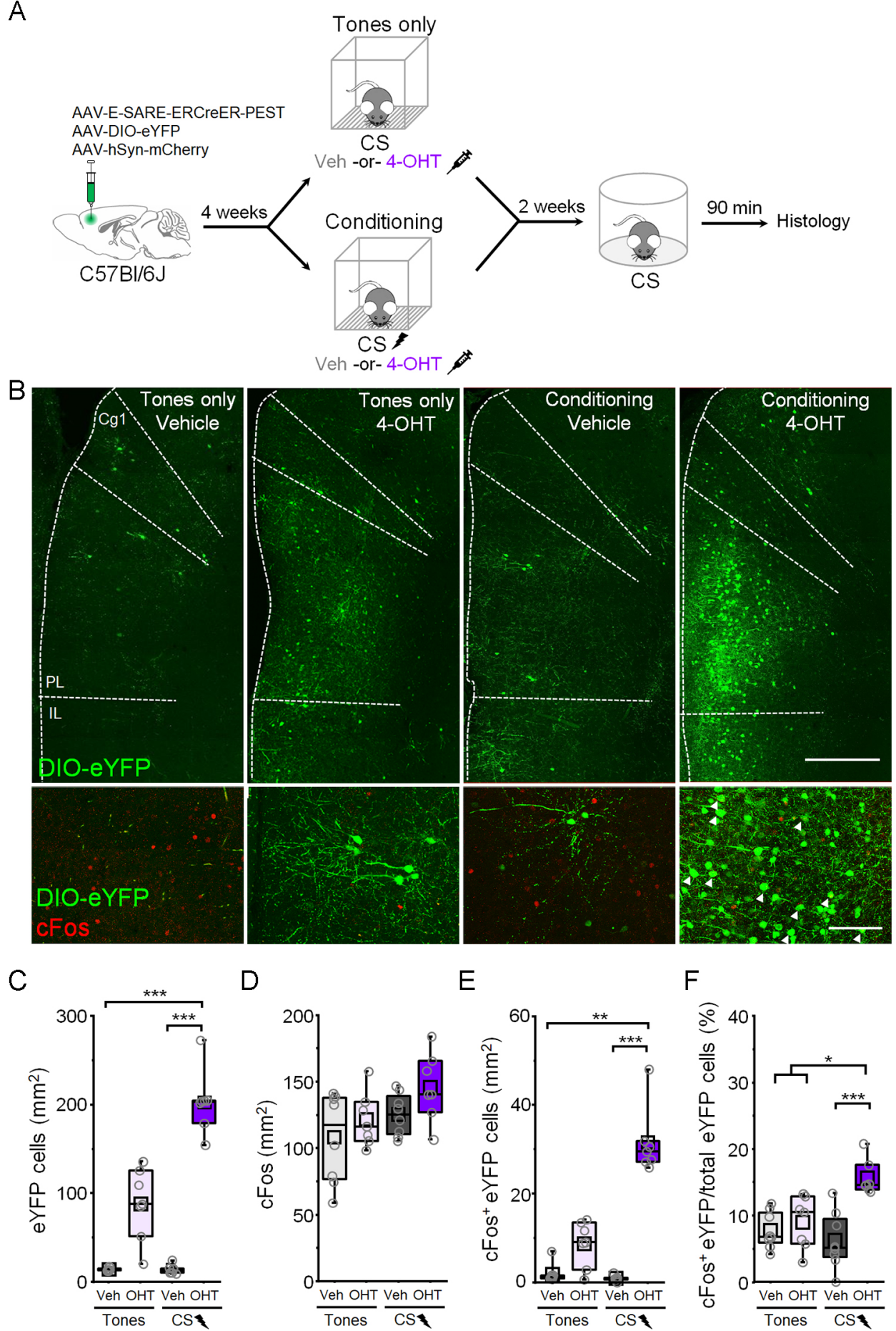

$E$

$\mathrm{F}$
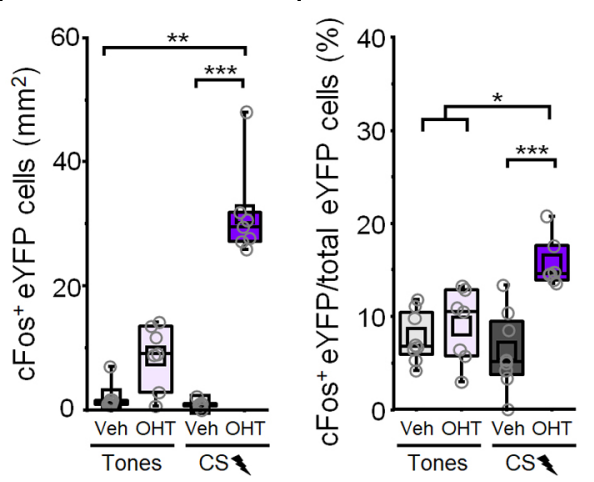

Figure 1. Fear conditioning recruits a prefrontal ensemble that is reactivated upon retrieval. (A) Wildtype (C57BI/6J) mice received bilateral infusions of a cocktail containing vectors encoding E-SARE-ERCreER, Cre-dependent eYFP, and hSyn-mCherry in $\mathrm{mPFC}$. Four weeks later, mice were either subjected to auditory tones (tones only) or CS-US pairing (conditioning) and then immediately injected with vehicle (veh) or 4-hydroxytamoxifen (4-OHT). Two weeks later, in a neutral context, all mice underwent a test of CS-evoked memory retrieval followed by cFos immunohistochemical analysis. (B) Top: representative histological images of eYFP tagging. Scale bar $=500 \mu \mathrm{m}$. Bottom: Induction of cFos following the retrieval test. White arrowheads denote eYFP tagged neurons immunoreactive for cFos. Scale bar $=100 \mu \mathrm{m}$. $\mathrm{Cg} 1=$ cingulate area 1 . $\mathrm{PL}=$ prelimbic cortex. IL = infralimbic cortex. (C-F) Comparison between tones only vehicle $(n=8$ mice), tones only 4-OHT $(n=7$ mice), conditioned vehicle $(n=8$ mice), and conditioned 4-OHT ( $n=7$ mice) groups of (C) number of eYFP+ cells: $\chi^{2}=23.69(3), p=2.89 \times 10^{-5}$, Kruskal-Wallis ANOVA; (D) number of cFos + cells: $\chi^{2}=5.75$ (3), $p=0.12$, Kruskal-Wallis ANOVA; (E) number of cFos+/eYFP+ double positive cells: $\chi^{2}=20.91(3), p=1.09 \times 10^{-4}$, Kruskal-Wallis ANOVA; and (F) number of cFos+/eYFP+ double positive cells normalized to the total number of eYFP+ cells: $\chi^{2}=16.73(3), p=8.05 x$ $10^{-4}$, Kruskal-Wallis ANOVA. Experiment was performed in 3 different cohorts and pooled together. ${ }^{*} p<0.05,{ }^{\star *} p<0.01,{ }^{* \star *} p<0.001$, Dunn's post hoc test (C-F). 
A

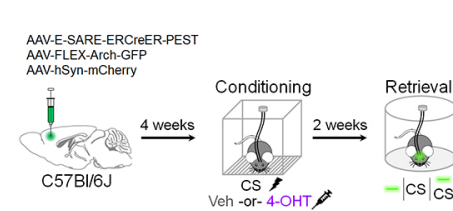

B
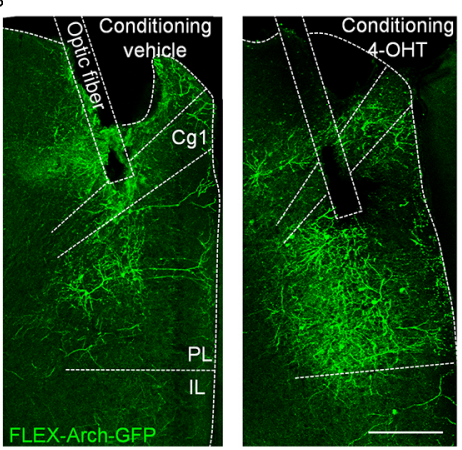

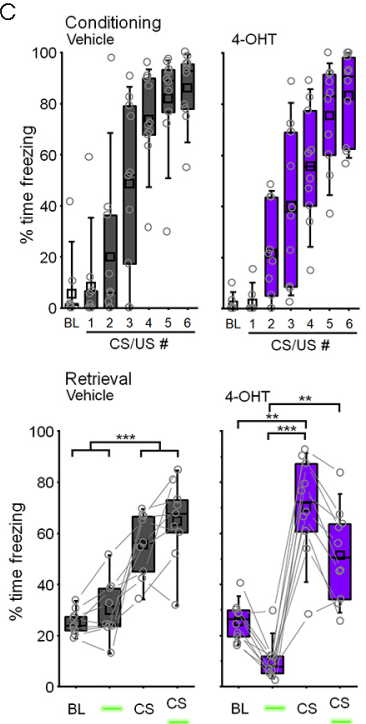

$\mathrm{BL}-\mathrm{CS}$ CS

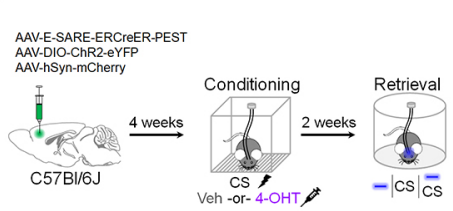

E
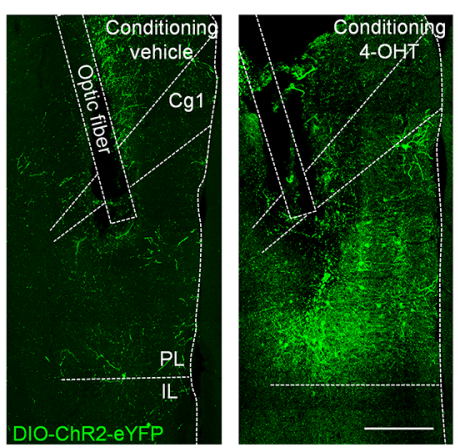

$\mathrm{F}$

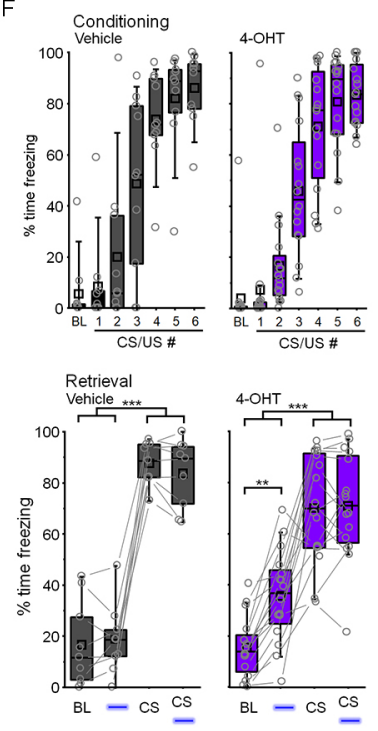

G

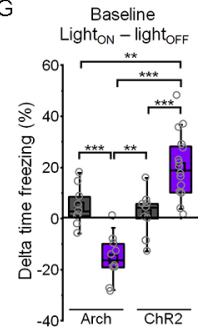

Figure 2. Prefrontal neurons activated by fear learning mediate conditioned freezing. (A) For in vivo optogenetic silencing of fear learning-related neurons, wildtype mice received bilateral infusions of a cocktail of vectors encoding E-SARE-ERCreER, Cre-dependent Arch, and hSyn-mCherry and were implanted with optic ferrules aimed at PL. Mice were subjected to CS-US pairing and immediately injected with vehicle (veh) or 4-hydroxytamoxifen (4-OHT). Freezing was quantified two weeks later in a neutral context in response to independent and combined light and CS trials. (B) Representative histological images of Arch expression and optic fiber placement. Scale $=500 \mu \mathrm{m}$. (C) Modulation of freezing by light (532 nm solid light, 20 s epochs) and CS trials in vehicle (gray) and 4-OHT (purple) injected mice. Vehicle: $F_{(1,8)}=408.43, p=3.75 \times 10^{-8}, 1$-way repeated measures ANOVA, $n=9$ mice. $4-O H T: \chi^{2}=27.84(3)$, $p=3.92 \times$ $10^{-6}$, Friedman ANOVA, $\mathrm{n}=10$ mice. Experiments were performed in 3 different cohorts and pooled together. (D) For in vivo optogenetic activation of fear learning-related neurons, wildtype mice received bilateral infusions of a cocktail of vectors encoding ESARE-ERCreER, Cre-dependent ChR2, and hSyn-mCherry and were implanted with optic ferrules aimed at PL. Behavior was conducted in a manner identical to (A). (E) Representative histological images of ChR2 expression and optic fiber placement. Scale = $500 \mu \mathrm{m}$. (F) Modulation of freezing by light ( $473 \mathrm{~nm}, 5 \mathrm{~ms}$ pulses, $20 \mathrm{~Hz}, 20 \mathrm{~s}$ epochs) and CS trials in vehicle (gray) and 4-OHT (purple) injected mice. Vehicle: $F_{(1,8)}=269.4, p=1.91 \times 10^{-7}, 1$-way repeated measures ANOVA, $n=9$ mice. 4-OHT: $F_{(1,15)}=196.72, p$ $=4.99 \times 10^{-10}$, 1-way repeated measures ANOVA, $n=16$ mice. Experiments were performed in 4 different cohorts and pooled together (G) Change in freezing induced by photostimulation during the baseline period in (C) and (F). Effect of photostimulation (LightonLightoff): $F_{(1,40)}=37.16, p=3.56 \times 10^{-7}$, interaction between opsin and light, 2-way ANOVA. (H) Change in freezing induced by photostimulation during CS trials in $(\mathbf{C})$ and $(\mathbf{F})$. Effect of photostimulation (Lighton- Light off $_{\text {): }} \mathrm{F}_{(1,40)}=14.45, \mathrm{p}=4.8 \times 10^{-4}$, interaction

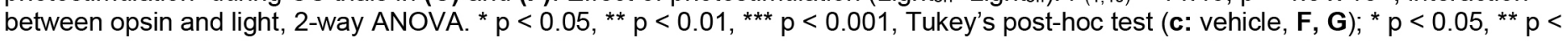
$0.01,{ }^{* * *} \mathrm{p}<0.001$, Dunn's post-hoc test $(\mathbf{c}: 4-\mathrm{OHT}) . \mathrm{Cg} 1=$ cingulate area $1 . \mathrm{PL}=$ prelimbic cortex. IL $=$ infralimbic cortex. 
A

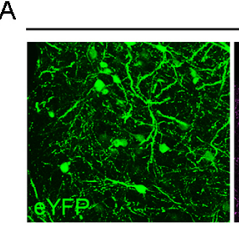

B

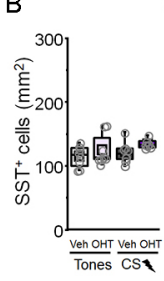

c
Somatostatin
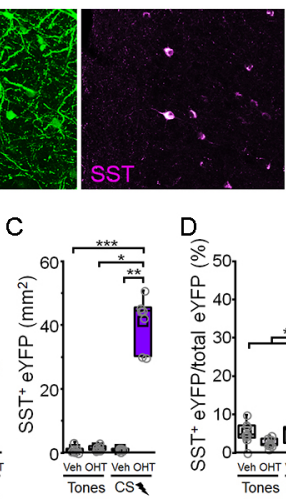
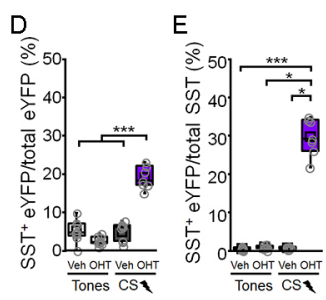

F

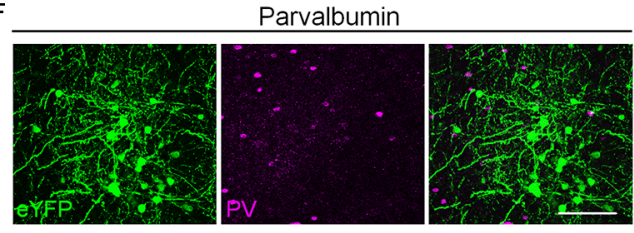

G

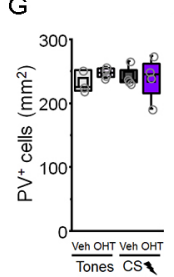

$\mathrm{H}$

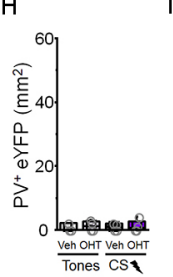

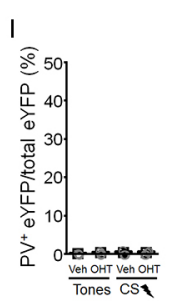

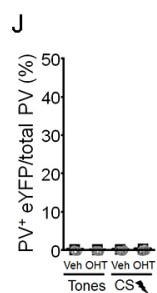

K

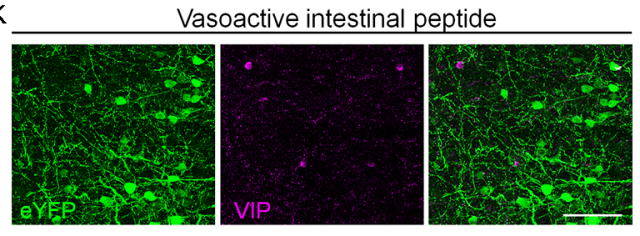

L

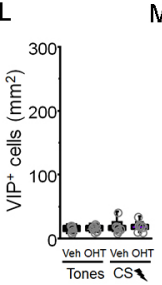

M

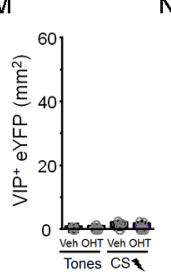

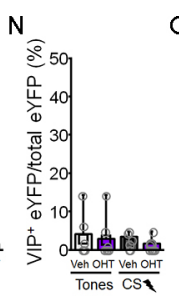

$\mathrm{O}$

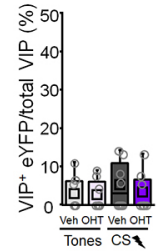

Figure 3. Fear learning-related ensembles are partly comprised of somatostatin-expressing interneurons. (A)

Immunohistochemical staining against somatostatin (SST) in prelimbic cortex tissue following CS-US pairing and 4-OHT injection. Scale $=50 \mu \mathrm{m}$. (B-E) Comparison between tones only vehicle $(n=8$ mice), tones only 4-OHT $(n=8$ mice), conditioning vehicle $(n=8$ mice), and conditioning 4-OHT ( $n=7$ mice) groups of $(B) S S T+$ cells, $\chi^{2}=6.934(3), p=0.074$, Kruskal-Wallis ANOVA; (C) SST+ eYFP cells, $\chi^{2}=16.9$ (3), $p=7.4 \times 10^{-4}$, Kruskal-Wallis ANOVA; (D) SST+ eYFP cells normalized to the total number of eYFP, F(1,27) $=87.91$, $p=5.55 \times 10^{-10}$, interaction between training and treatment, 2-way ANOVA, and (E) SST+ eYFP cells normalized to the total number of SST+ cells per group, $\chi^{2}=17.04$ (3), $p=6.93 \times 10^{-4}$, Kruskal-Wallis ANOVA. (F) Immunohistochemical staining against parvalbumin (PV) in prelimbic cortex tissue following CS-US pairing and 4-OHT injection. Scale $=50 \mu \mathrm{m}$. (G-J) Comparison between tones only vehicle $(n=3)$, tones only 4-OHT $(n=4)$, conditioning vehicle $(n=4)$, and conditioning 4-OHT $(n=4)$ groups of $(G) P V+$ cells, $F(1,40)=$ $0.73, p=0.41$, 2-way ANOVA; (H) PV+ eYFP cells, $\chi^{2}=0.485$ (3), $p=0.922$, Kruskal-Wallis ANOVA; (I) PV+ eYFP cells normalized to the total number of eYFP+ cells per group, $\chi^{2}=0.882(3), p=0.829$, Kruskal-Wallis ANOVA; and (J) PV+ eYFP cells normalized to the total number of $P V+$ cells per group, $\chi^{2}=1.31(3), p=0.727$, Kruskal-Wallis ANOVA. (K) Immunohistochemical staining against vasoactive intestinal peptide (VIP) in prelimbic cortex tissue following CS-US pairing and 4-OHT injection. Scale $=50 \mu \mathrm{m}$. $(\mathrm{L}-\mathrm{O})$ Comparison between tones only vehicle $(n=8)$, tones only 4-OHT $(n=8)$, conditioning vehicle $(n=8)$, and conditioning 4-OHT $(n=8)$ groups of (L) VIP+ cells, $\chi^{2}=1.84(3), p=0.606$, Kruskal-Wallis ANOVA; (M) VIP+ eYFP cells, $\chi^{2}=1.56(3)$, $p=0.668$, Kruskal-Wallis ANOVA; (N) VIP+ eYFP cells normalized to the total number of eYFP+ cells per group, $\chi^{2}=0.359(3), p=0.949$, Kruskal-Wallis ANOVA; and (O) VIP+ eYFP cells normalized to the total number of VIP+ cells per group, $\chi^{2}=1.02(3), p=0.796$, Kruskal-Wallis ANOVA. Experiments were performed in 2 different cohorts and pooled together. ${ }^{\star \star *} p<0.001$, Tukey's post-hoc test (D). ${ }^{\star} p<0.05$, ${ }^{\star *}$ $\mathrm{p}<0.01,{ }^{* * *} \mathrm{p}<0.001$, Dunn's post-hoc test $(\mathbf{C}, \mathbf{E})$. 
bioRxiv preprint doi: https://doi.org/10.1101/2021.07.18.452791; this version posted July 19, 2021. The copyright holder for this preprint (which was not certified by peer review) is the author/funder, who has granted bioRxiv a license to display the preprint in perpetuity. It is made available under aCC-BY-NC-ND 4.0 International license.

A
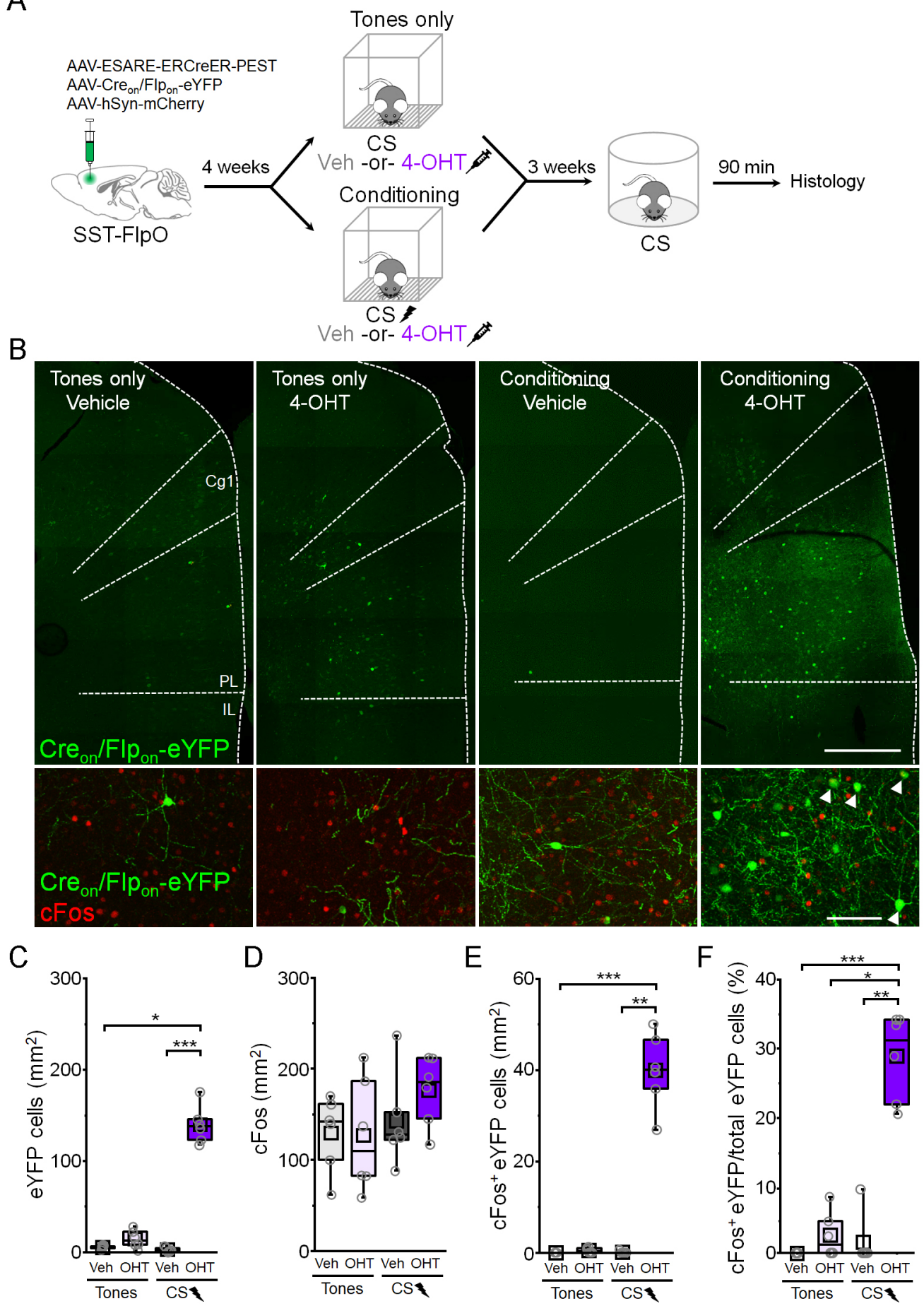

Figure 4: Fear learning-activated somatostatin-interneurons are preferentially reactivated upon memory retrieval. (A) SSTFlpO transgenic mice received bilateral infusions into prelimbic cortex of a cocktail containing vectors encoding E-SARE-ERCreER, Cre- and Flp-dependent eYFP, and hSyn-mCherry. Four weeks later, mice received either CS-US pairing (conditioning) or auditory tones (tones only) and were then immediately injected with vehicle (veh) or 4-hydroxytamonifen (4-OHT). After an additional three weeks, in a neutral context, all mice underwent a test of CS-evoked memory retrieval followed by cFos immunohistochemical analysis. (B) Top: representative histological images of eYFP tagging. Scale bar $=500 \mu \mathrm{m}$. Bottom: Induction of cFos following the retrieval test. White arrowheads denote eYFP neurons immunoreactive for cFos. Scale bar $=100 \mu \mathrm{m}$. Cg1 = cingulate area 1. PL $=$ prelimbic cortex. $\mathrm{IL}=$ infralimbic cortex. (C-F) Comparison between tones only vehicle $(n=6$ mice), tones only 4-OHT $(n=6$ mice), conditioning vehicle ( $n=6$ mice), and conditioning 4-OHT ( $n=6$ mice) groups of (C) number of eYFP+ cells: $\chi^{2}=17.65(3), p=5.2 \times 10^{-4}$, Kruskal-Wallis ANOVA; (D) number of cFos+ cells: $F_{(1,20)}=0.82, p=0.376,2$-way ANOVA; $(E)$ number of cFos+/eYFP+ double positive cells: $x^{2}=$ $17.97(3), p=4.46 \times 10^{-4}$, Kruskal-Wallis ANOVA; and (F) number of cFos+/eYFP+ double positive cells normalized to the total number of eYFP+ cells in each group: $x^{2}=17.53(3), p=5.49 \times 10^{-4}$, Kruskal-Wallis ANOVA. Experiment was performed in 4 different cohorts and pooled together. ${ }^{*} p<0.05,{ }^{* *} p<0.01,{ }^{* * *} p<0.001$, Dunn's post-hoc test $(\mathbf{C}, \mathrm{E}, \mathrm{F})$. 
A

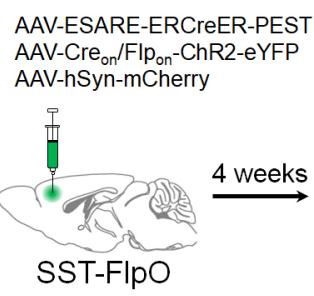

C
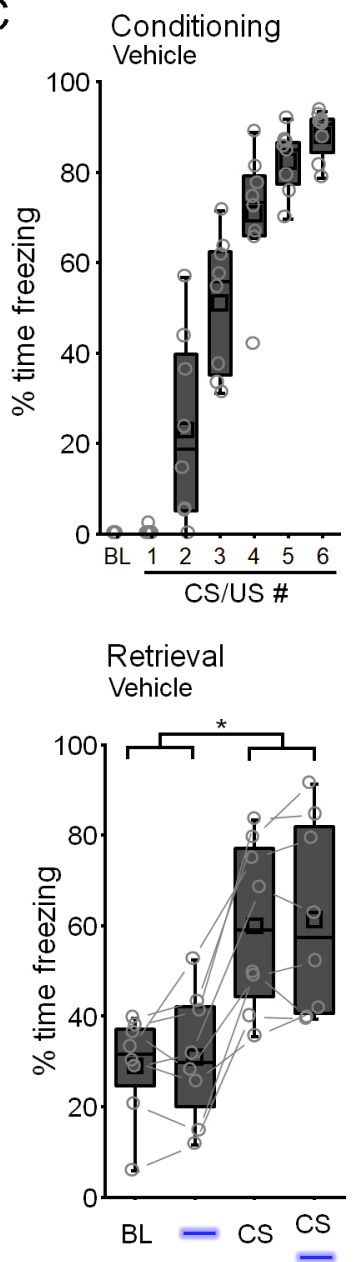

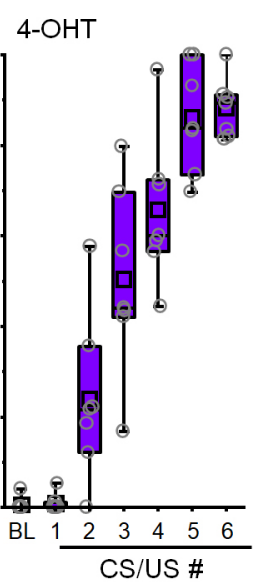

$4-\mathrm{OHT}$

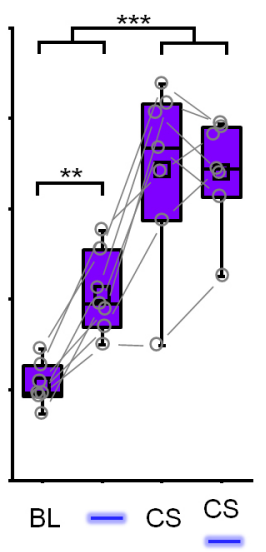

Figure 5: Selective stimulation of learning-activated somatostatin interneurons elicits freezing. (A) For in vivo optogenetic activation of fear learning-related SST-INs, SST-FIpO transgenic mice received bilateral infusions of a cocktail of vectors encoding ESARE-ERCreER, Cre- and Flp-dependent ChR2, and hSyn-mCherry and were implanted with optic ferrules aimed at PL. Mice were subjected to CS-US pairing and immediately injected with vehicle (veh) or 4-hydroxytamoxifen (4-OHT). Freezing was quantified three weeks later in a neutral context while testing the independent and combined effect of light and CS trials. (B) Representative histological images of ChR2 expression and optic fiber placement. Scale $=500 \mu \mathrm{m}$. Cg1 $=$ cingulate area $1 . \mathrm{PL}=$ prelimbic cortex. IL $=$ infralimbic cortex. (C) Modulation of freezing by light $(473 \mathrm{~nm}, 10 \mathrm{~ms}$ pulses, $20 \mathrm{~Hz}, 20$ s epochs) and CS trials in vehicle (gray) and 4-OHT (purple) injected mice. Vehicle: $\chi^{2}=19.5(3), p=2.15 \times 10^{-4}$, Friedman ANOVA, $n=8$ mice. 4-OHT: $F_{(3,18)}=46.7, p=1.07 \times 10^{-8}, 1-$ way repeated measures ANOVA, $n=7$ mice. Experiment was performed in 2 different cohorts and pooled together. ${ }^{\star \star} p<0.01,{ }^{\star \star \star} p<$ 0.001 , Tukey's post-hoc test $(\mathbf{C}: 4-\mathrm{OHT}) .{ }^{*} p<0.05$, Dunn's post-hoc test (C: vehicle). 
bioRxiv preprint doi: https://doi.org/10.1101/2021.07.18.452791; this version posted July 19, 2021. The copyright holder for this preprint (which was not certified by peer review) is the author/funder, who has granted bioRxiv a license to display the preprint in perpetuity. It is made available under aCC-BY-NC-ND 4.0 International license.

A

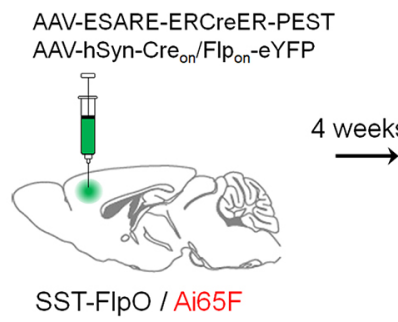

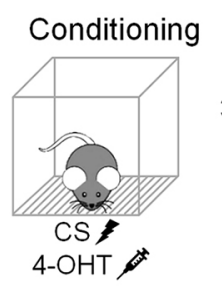

C

B
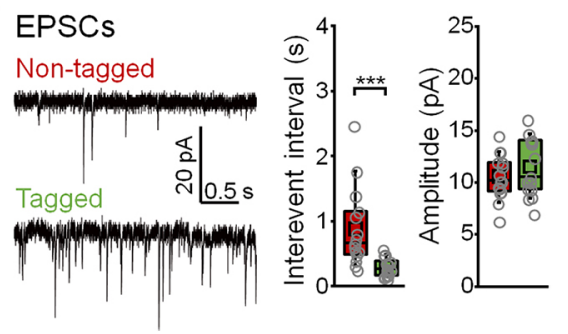

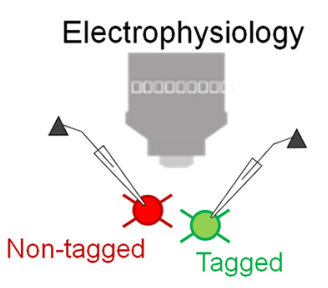

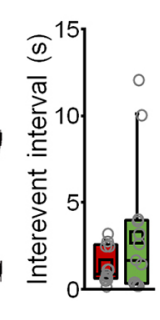

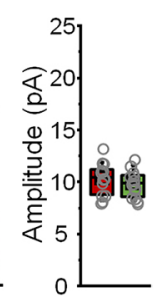

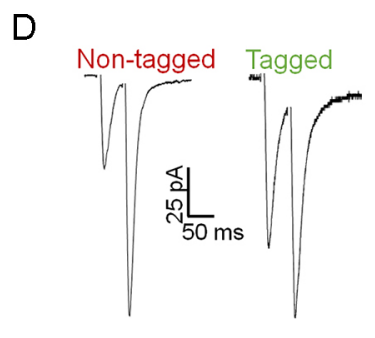

E

G

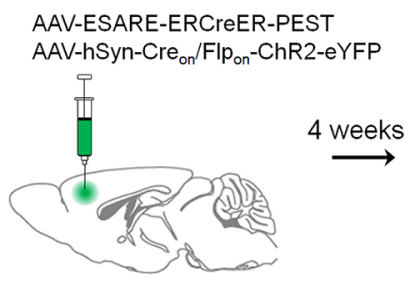

SST-FIpO/ PV-Cre/ Ai9
Conditioning
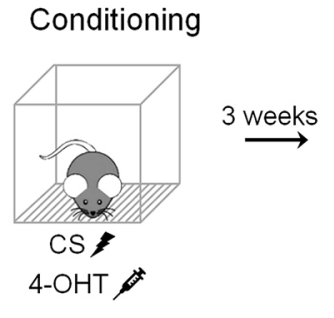
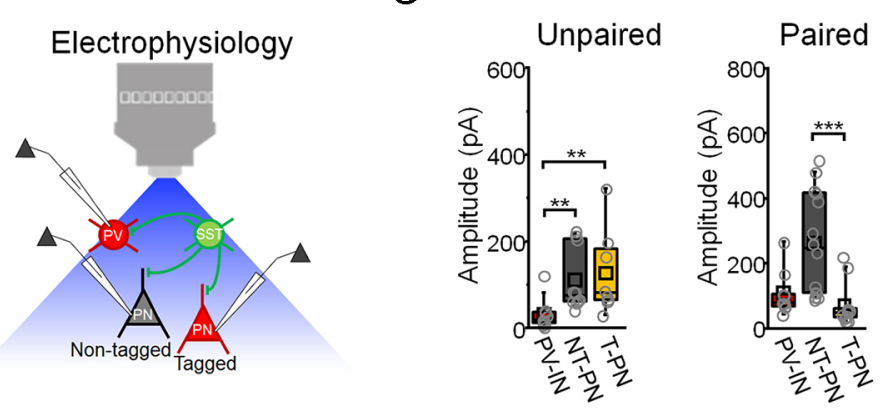

F
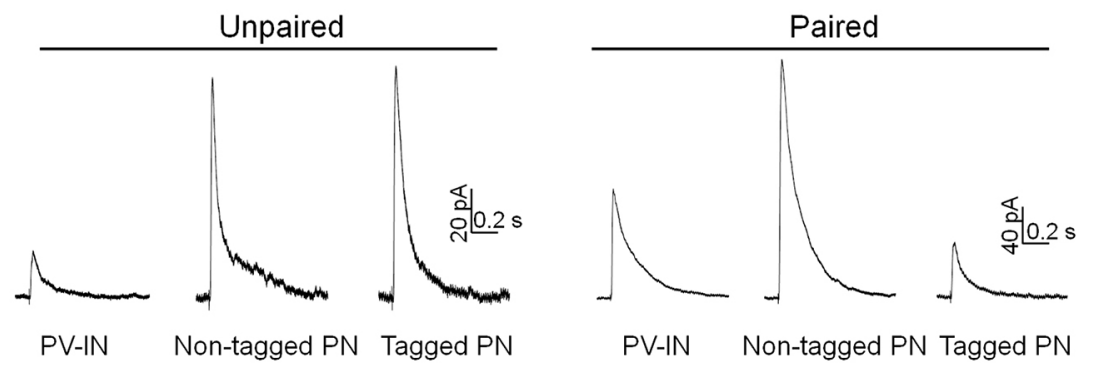

$\mathrm{H}$

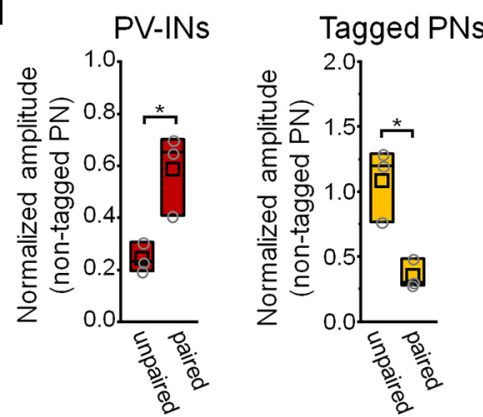

Figure 6: Input and output synaptic connections of learning-activated somatostatin interneurons exhibit distinct properties. (A) To independently target and record from both tagged and non-tagged SST-INs, which represent activated and non-activated populations, we infused into the prelimbic cortex of SST-FIpO/ Ai65F double transgenic mice a cocktail of vectors encoding E-SAREERCreER, as well as Cre- and Flp-dependent eYFP. Mice were subjected to CS-US pairing and immediately injected with 4hydroxytamoxifen (4-OHT). Three weeks later, recordings were obtained from eYFP+/ tdTomato+ (tagged) and eYFP-/ tdTomato+ (non-tagged) SST-INs. (B) Spontaneous excitatory postsynaptic currents (EPSCs) were collected by interleaved recordings from tagged ( $n=15$ cells) and non-tagged SST-INs $(n=15$ cells) in the same slices ( $n=6$ slices from 5 mice). Interevent interval: $U=205, p$ $=1.35 \times 10^{-4}$, Mann-Whitney U-test. Amplitude: $t_{28}=-1.03, p=0.31$, two-sided unpaired t-test. (C) Spontaneous inhibitory postsynaptic currents (IPSCs) were recorded from tagged ( $n=14$ cells) and non-tagged SST-INs $(n=14$ cells) in the same slices $(n=6$ slices from 5 mice). Interevent interval: $U=89, p=0.696$, Mann-Whitney U-test. Amplitude: $t_{26}=0.945, p=0.353$, Mann-Whitney U-test. (D) EPSC recordings from tagged ( $n=10$ cells) and non-tagged SST-INs $(n=11$ cells $)$ in the same slices $(n=4$ slices in 3 mice) during paired pulse stimulation. Paired pulse ratio: $F_{(3,7)}=16.64, p=0.0014$, interaction between cell type and delay, 2-way repeated measures ANOVA. (E) To isolate GABAergic responses at connections from tagged SST-INs onto PV-INs as well as tagged and non-tagged PNs, SST-FIpO/ PV-IRES-Cre/ Ai9 triple transgenic mice received infusions of a cocktail of vectors encoding E-SARE-ERCreER, as well as Cre- and Flp-dependent ChR2. Mice were subjected to either CS-US pairing or unpaired conditioning and injected with 4-OHT. Three weeks later, recordings were obtained from tdTomato+ PV-INs as well as tagged (tdTomato+) and non-tagged (td-Tomato-) PNs. (F) Example IPSC traces. (G) Amplitude of IPSCs resulting from photoexcitation ( $460 \mathrm{~nm}, 1 \mathrm{~ms}, 0.1 \mathrm{~Hz})$ of tagged SST-INs in PV-INs, nontagged PNs (NT-PNs), and tagged PNs (T-PNs) following unpaired conditioning ( $n=3$ slices from 3 mice: PV-INs $(n=10$ cells), NTPNs $(n=10$ cells), T-PNs $(n=8$ cells $)$ ) and CS-US pairing $(n=3$ slices from 3 mice: PV-INs $(n=9$ cells $)$, NT-PNs $(n=14$ cells $)$, T-PNs $(n=12$ cells $)$ ). Unpaired conditioning: $x^{2}=12.95(2), p=0.0015$, Kruskal-Wallis ANOVA. CS-US pairing: $x^{2}=17.85(2), p=1.32 \times 10^{-4}$, Kruskal-Wallis ANOVA. (H) IPSC amplitudes of PV-INs and tagged PNs normalized to the median amplitude of non-tagged PNs from the same slices. PV-IN amplitude: $t_{4}=3.56, p=0.023$, two-sided unpaired t-test. Tagged PN amplitude: $t_{4}=4.19, p=0.013$, two-sided unpaired t-test. ${ }^{*} p<0.05,{ }^{* *} p<0.01,{ }^{* * *} p<0.001$ by Mann Whitney U-test (B), Tukey's post-hoc test (D), unpaired t-test (H) or Dunn's post-hoc test (G). 
bioRxiv preprint doi: https://doi.org/10.1101/2021.07.18.452791; this version posted July 19, 2021. The copyright holder for this preprint (which was not certified by peer review) is the author/funder, who has granted bioRxiv a license to display the preprint in perpetuity. It is made available under aCC-BY-NC-ND 4.0 International license.

A

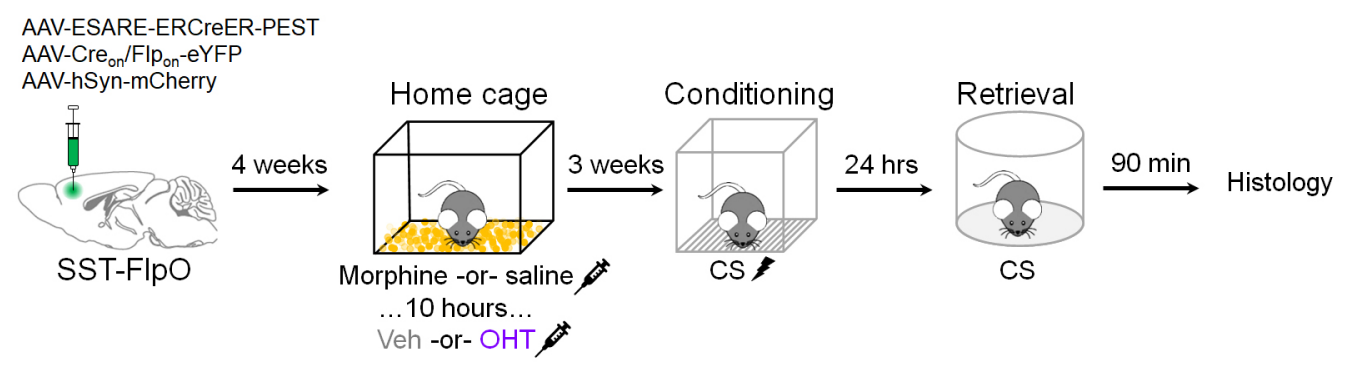

B
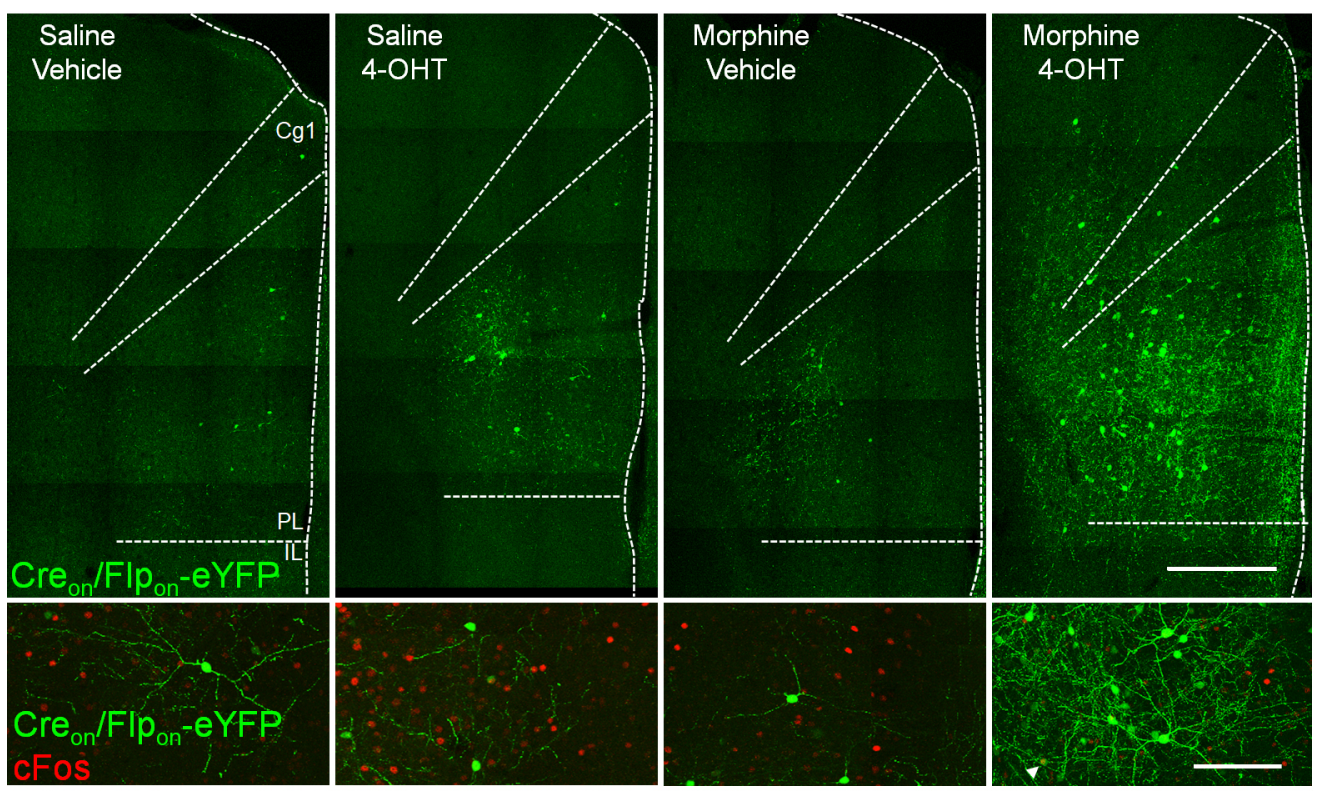

C

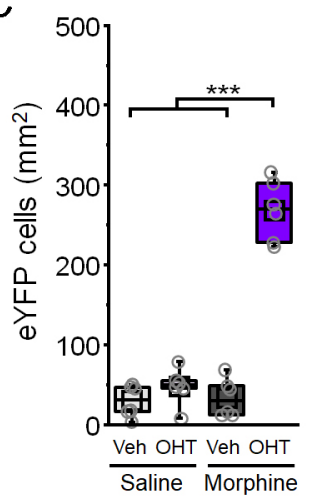

$\mathrm{D}$

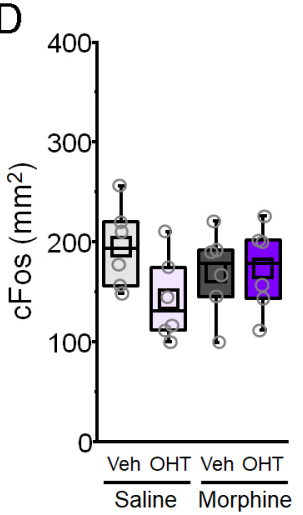

$\mathrm{E}$

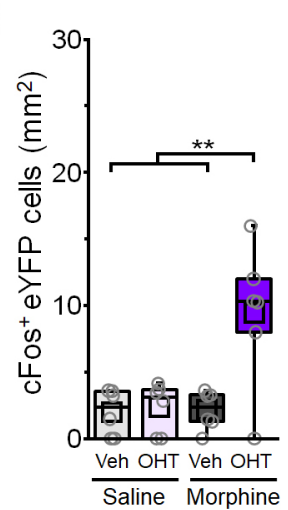

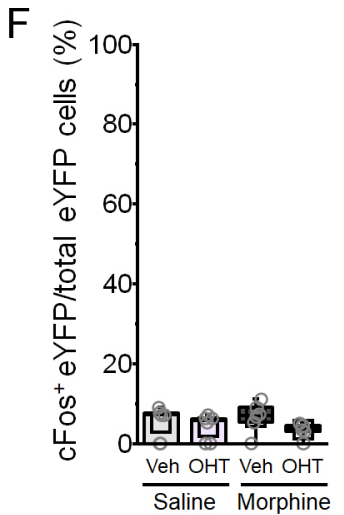

Figure 7: Morphine treatment activates a population of somatostatin-interneurons orthogonal to those involved in fear memory retrieval. (A) SST-FlpO transgenic mice received bilateral infusions into prelimbic cortex of a cocktail containing vectors encoding E-SARE-ERCreER, Cre- and Flp-dependent eYFP, and hSyn-mCherry. Four weeks later, in the home cage, they were injected with saline or morphine $(10 \mathrm{mg} / \mathrm{kg}$ ) followed 10 hours later by injections of vehicle (veh) or 4-hydroxytamoxifen (4-OHT). After another 3 weeks, they underwent auditory fear conditioning followed 24 hours later by a test of CS-evoked memory retrieval and cFos immunochemical analysis. (B) Top: representative histological images of SST-IN tagging for mice injected with saline and morphine and subsequently injected with either vehicle or 4-OHT. Scale bar $=500 \mu \mathrm{m}$. Bottom: Induction of cFos following CS-evoked memory retrieval. White arrowhead denotes cFos + tagged SST-IN. Scale bar $=100 \mu \mathrm{m} . \mathrm{Cg} 1=$ cingulate area $1 . \mathrm{PL}=$ prelimbic cortex. IL $=$ infralimbic cortex. (C-F) Comparison between saline vehicle $(n=6$ mice), tones only 4-OHT $(n=6$ mice), conditioning vehicle $(n=6$ mice), and conditioning 4-OHT ( $n=6$ mice) groups of $(C)$ number of eYFP+ cells: $F_{(1,20)}=96.09, p=4.418 \times 10^{-9}$, interaction between drug and treatment, 2-way ANOVA; (D) number of $c F o s+$ puncta: $F_{(1,20)}=2.62, p=0.121,2$-way ANOVA; (E) number of $c F o s+/ e Y F P+$ double positive cells: $F_{(1,20)}=7.77, p=0.011$, interaction between drug and treatment, 2-way ANOVA; and $(F)$ number of cFos+leYFP+ double positive cells normalized to the total number of eYFP+ cells in each group: $\chi^{2}=4.95(3), p=0.175$, Kruskal-Wallis ANOVA. ${ }^{* *} p$ $<0.01,{ }^{* * *} p<0.001$, Tukey's post-hoc test $(\mathbf{C}, \mathbf{E})$. 
bioRxiv preprint doi: https://doi.org/10.1101/2021.07.18.452791; this version posted July 19, 2021. The copyright holder for this preprint (which was not certified by peer review) is the author/funder, who has granted bioRxiv a license to display the preprint in perpetuity. It is made available under aCC-BY-NC-ND 4.0 International license.

\section{A}

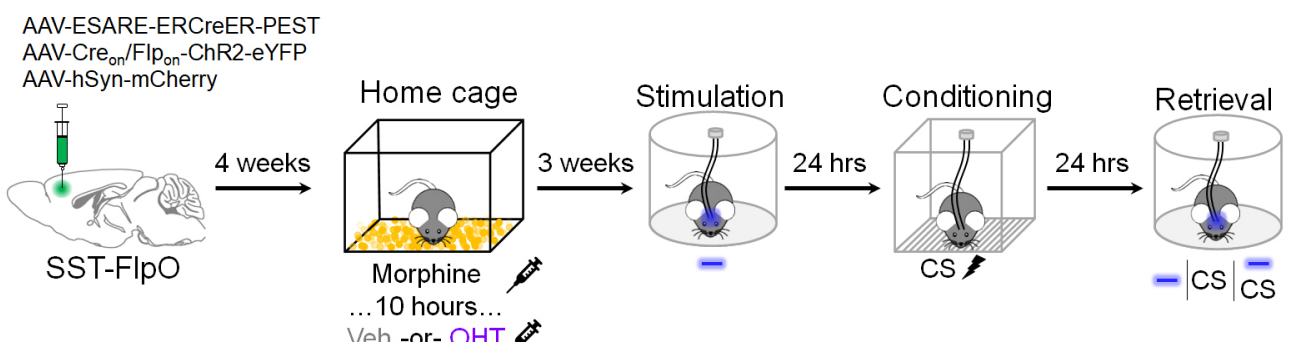

B
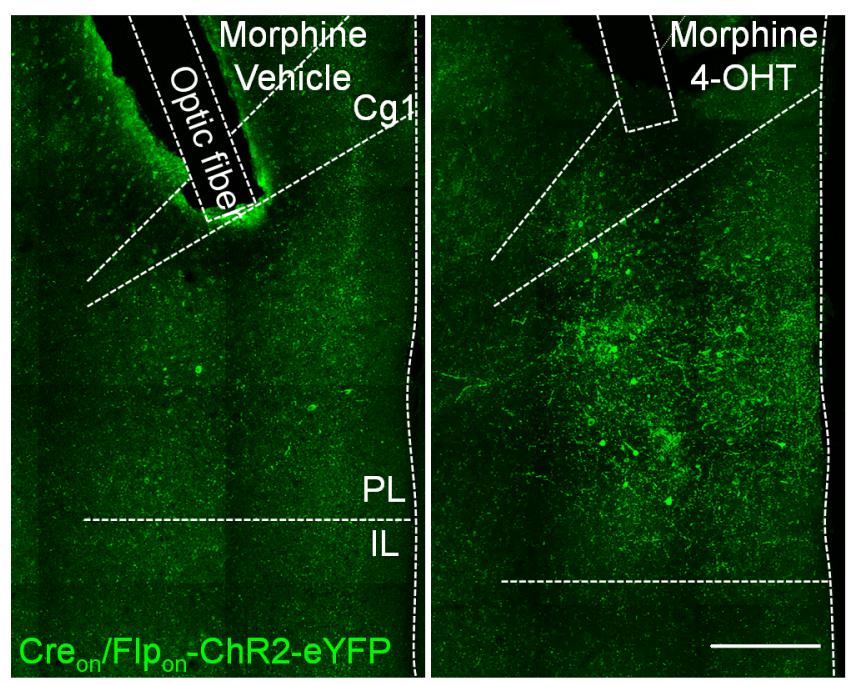

C

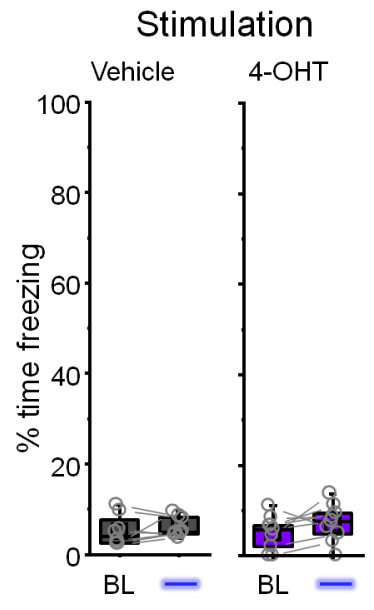

$\mathrm{D}$

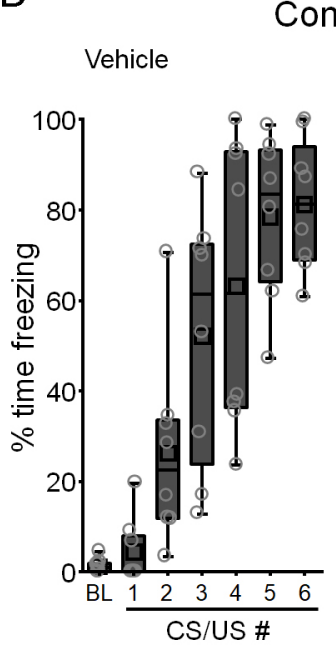

Conditioning

4-OHT

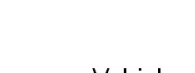

Retrieval
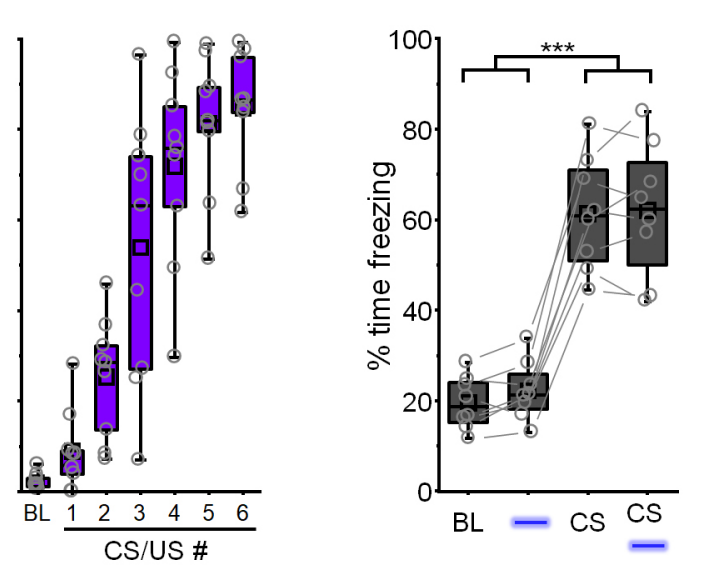

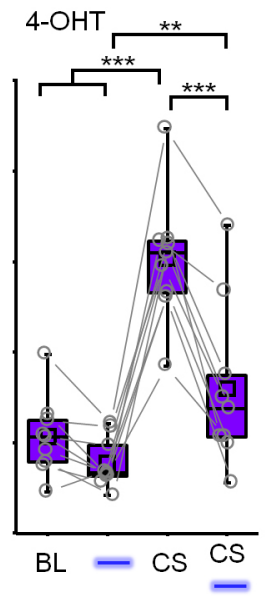

Figure 8: Somatostatin-interneurons activated by morphine oppose conditioned fear expression. (A) For in vivo optogenetic activation of morphine-related SST-INs, SST-FlpO transgenic mice received bilateral infusions into prelimbic cortex of a cocktail of vectors encoding E-SARE-ERCreER, Cre- and Flp-dependent ChR2, and hSyn-mCherry and were implanted with optic ferrules aimed at PL. Mice received an injection of morphine $(10 \mathrm{mg} / \mathrm{kg})$ and 10 hours later, injections of vehicle (veh) or 4-hydroxytamoxifen (4-OHT). Photostimulation-induced freezing (Stimulation) was quantified three weeks later in a neutral context. Mice were then subjected to CSUS pairing (Conditioning) and 24 hours later, a CS-evoked memory retrieval test (Retrieval) in a neutral context, where the independent and combined effects of light and CS trials were quantified. (B) Representative histological images of ChR2 expression and optic fiber placement. Scale $=500 \mu \mathrm{m} . \mathrm{Cg} 1=$ cingulate area 1. $\mathrm{PL}=$ prelimbic cortex. IL $=$ infralimbic cortex. (C) Modulation of freezing by photoexcitation $\left(473 \mathrm{~nm}, 10 \mathrm{~ms}\right.$ pulses, $20 \mathrm{~Hz}, 20 \mathrm{~s}$ epochs) in vehicle (gray) and 4-OHT (purple) mice. Vehicle: $t_{7}=-0.788, p=0.456$, paired t-test. 4-OHT: $t_{8}=-1.74, p=0.119$, paired t-test. (D) Modulation of freezing by photoexcitation $(473 \mathrm{~nm}, 10 \mathrm{~ms}$ pulses, $20 \mathrm{~Hz}, 20$ $s$ epochs) and CS presentation during the retrieval test for vehicle (gray) and 4-OHT (purple) mice. Vehicle: $F_{(3,21)}=109.48, p=5.54 x$ $10^{-13}, 1$-way repeated measures ANOVA. 4-OHT: $F_{(3,24)}=46.69, p=3.59 \times 10^{-10}, 1$-way repeated measures ANOVA. ${ }^{* *} p<0.01,{ }^{* * *} p<$ 0.001 , Tukey's post-hoc test (D). 